\title{
Deflation-accelerated preconditioning of the Poisson-Neumann Schur problem on long domains with a high-order discontinuous element-based collocation method
}

\author{
Sumedh M. Joshi \\ Center for Applied Mathematics, \\ 657 Rhodes Hall, Cornell University, \\ Ithaca $N Y, 14850$ \\ Greg N. Thomsen \\ The University of Texas at Austin \\ Austin TX, 78712 \\ Peter J. Diamessis \\ School of Civil and Environmental Engineering, \\ 105 Hollister Hall, Cornell University, \\ Ithaca $N Y, 14850$
}

\begin{abstract}
A combination of block-Jacobi and deflation preconditioning is used to solve a high-order discontinuous element-based collocation discretization of the Schur complement of the Poisson-Neumann system as arises in the operator splitting of the incompressible Navier-Stokes equations. The preconditioners and deflation vectors are chosen to mitigate the effects of ill-conditioning due to highly-elongated domains typical of simulations of strongly non-hydrostatic environmental flows, and to achieve GMRES convergence independent of the size of the number of elements in the long direction. The ill-posedness of the Poisson-Neumann system manifests as an inconsistency of the Schur complement problem, but it is shown that this can be accounted for with appropriate projections out of the null space of the Schur complement matrix without affecting the accuracy of the solution. The block-Jacobi preconditioner is shown to yield GMRES convergence independent of the polynomial order and only weakly dependent on the number of elements within a subdomain in the decomposition. The combined deflation and block-Jacobi preconditioning is compared with two-level non-overlapping block-Jacobi preconditioning of the Schur problem, and while both methods achieve convergence independent of the grid size, deflation is shown to require half as many GMRES iterations and $25 \%$ less wall-clock time for a variety of grid sizes and domain aspect ratios. The deflation methods shown to be effective for the two-dimensional Poisson-Neumann problem are extensible to the three-dimensional problem assuming a Fourier discretization in the third dimension. A Fourier discretization results in a two-dimensional Helmholtz problem for each Fourier component that is solved using deflated block-Jacobi preconditioning on its Schur complement. Here again deflation is shown to be superior to two-level non-overlapping block-Jacobi preconditioning, requiring about half as many GMRES iterations and $15 \%$ less time.
\end{abstract}

Keywords: Poisson equation, spectral element, deflation, preconditioning, Schur complement, domain decomposition

\section{Introduction}

When operator-splitting the incompressible Navier-Stokes equations in the context of computational fluid dynamics, the satisfaction of the divergence-free condition on the velocity requires the solution of a Poisson equation with Neumann boundary conditions [39. This pressure Poisson equation plays the role

*Please send correspondence to the first author at smj96@cornell.edu 
of enforcing incompressibility of the velocity field, and computing its accurate solution is the dominant computational expense in numerically solving the incompressible Navier-Stokes equations 21. This paper describes a method for solving the Poisson-Neumann problem 17

$$
\begin{aligned}
\nabla^{2} u & =f \text { on } \Omega \\
n \cdot \nabla u & =g \text { on } \partial \Omega,
\end{aligned}
$$

as it arises in the context of solving the incompressible Navier-Stokes equations on element-based discrete grids in environmental fluid dynamics of strongly non-hydrostatic flow phenomena where the pressure cannot be computed by simply integrating the hydrostatic balance in the vertical. Due to the fact that any constant function added to a solution $u$ is also a solution, this problem is ill-posed [50], and so a compatibility condition,

$$
\int_{\Omega} f d \Omega=\int_{\partial \Omega} g d S
$$

must be added to make the system solvable.

The simulation of non-hydrostatic environmental phenomena have is uniquely challenging from a computational perspective. While the discrete Poisson problem in general has certainly garnered much attention in the literature, the long, high-aspect ratio domains in non-hydrostatic environmental flows add difficulties that can be characterized as follows. First, to avoid artificial numerical dispersion and dissipation that can pollute a low-order discretization 59, 17, high-order discretizations are required for accurately computing dynamical properties of environmental flows with reasonable computational effort. These high-order methods are more difficult to implement in practice, and they can result in ill-conditioned operator matrices that must be carefully constructed to avoid numerical error [14. Secondly, due to the presence of gravity, environmental problems often have very different spatial and temporal scales in the vertical direction than in the horizontal direction. Thus, computational grids tend to be highly anisotropic with elements frequently compressed in the vertical direction and greatly elongated in the horizontal direction [54, 53, 60. Such anisotropic grids and high-aspect ratio domains arise, for example, in the simulation of the shoaling of internal gravity over sloping bathymetry [42], in modeling of the internal dynamics of estuarial flows [29], or studying the nature of turbulence in stratified wakes [63, 19].

One approach to addressing the particular difficulties in non-hydrostatic environmental flows is to exploit physical properties to tailor a numerical method. Asymptotic expansions in the leptic (aspect) ratio of the grid are sometimes used to divide the Poisson operator into two coupled problems, one in the vertical (thin) direction, and another problem in the remaining dimensions [54]. These methods have been successfully combined with geometric multi-grid [53] and Krylov methods [52] for obtaining the solution to the independent sub-problems derived in the leptic expansion. Yet another physical approach is to formulate the Poisson problem in a coordinate system following the perturbations of the density iso-contours of the flow field. By making a small-slope approximation of the iso-contours, a simpler Poisson problem is solved. This method has been shown to be effective in reproducing the dynamics of nonlinear internal wave propagation at the environmental scale [60, but cannot capture more localized multiscale phenomena such as turbulence and the nonlinear stages of evolution of a two-dimensional instability.

Apart from such physically-inspired approaches, ideas from domain decomposition can also be used to address the difficulty of solving high-order long-domain discretizations at the algebraic level. While domain decomposition methods are general, they have properties that are beneficial for the ill-conditioned Poisson-Neumann problem on stretched grids. Since most environmental flow problems tend to be too large for direct matrix factorization, domain decomposition methods focus on constructing effective preconditioners for the iterative solvers. Efforts have been made to alleviate the ill-conditioning of the element matrices due to aspect-ratio, as well as to yield convergence of iterative solvers independent of the size of the number of elements in the grid [27, 25. Since in environmental problems domains have high aspect ratio and often contain many elements, domain decomposition is well-suited for this application.

In this work, domain decomposition is used to construct decoupled local problems and a Schur complement system that, while smaller and better numerically conditioned than the full problem, is still generally too large in practice to solve directly. Thus the solution of the Schur complement problem is

\footnotetext{
${ }^{1}$ in which the real-valued function $u(x, z)$ represents the pressure.
} 
usually obtained iteratively. The Schur complement approach to solving the elliptic equations has been implemented for high-order [15] spectral and collocation-based [5] discretizations with iterative Krylov solvers. As is common with Krylov methods, the Schur complement approach ubiquitously requires an effective preconditioner to be practical. A modern class of domain-decomposition based preconditioners are the multilevel additive-Schwarz methods, and they have been shown to be very effective on the Schur complement problem of the Helmholtz [43, 48, and the Poisson-Dirichlet equations [49].

Closely related to multi-level additive-Schwarz methods, deflation methods accelerate the convergence of Krylov methods by attempting to identify and directly solve components of the residual associated with slowly-converging eigenvectors [61. Deflation methods have been shown to be superior to two-level block-Jacobi methods for fluid-flow problems with symmetric positive-definite discretizations [45, but, to the knowledge of the authors, have not been compared with block-Jacobi methods for the Schur complement problem, nor been applied to environmental flow problems. However, deflation methods have been shown to be effective for the Poisson-Neumann problem [57] and so it is reasonable to expect deflation to perform well for the Schur complement problem as well. In this paper, it is demonstrated that on high-order, high-aspect ratio grids deflation augmented with block-Jacobi preconditioning achieves convergence of the Poisson-Neumann Schur system independent of both the aspect ratio and the number of elements in the long direction. Furthermore it is shown that deflated block-Jacobi preconditioning is at least as effective as the two-level block-Jacobi method, requiring approximately half as many Krylov iterations as the two-level block-Jacobi preconditioning method. While this is demonstrated with the Spectral Multidomain Penalty Method [17] discretization, the ideas outlined herein may be applied to any high-order discontinuous element-based discretization of an elliptic problem, which encompasses a broad and important class of methods and partial differential equations.

The paper is organized as follows. In Section 2 is an introduction of the discretization of PoissonNeumann problem used here along with the construction of its Schur complement problem. In Section 3 is a section of the deflated accelerated block-Jacobi preconditioning method for solving the Schur complement system. In Section 4 are the results comparing the performance of deflation augmented preconditioning with two-level block-Jacobi preconditioning on a suite of test problems. In Section 5 is shown an extension of this method to the three-dimensional problems. Section 6 is a discussion of the broader applicability of the results, and Section 7 is a summary of the results and concluding remarks.

\section{Construction of the Schur complement problem}

\subsection{Background on Schur complement methods}

Schur complement methods for partial differential equations arise in many contexts related to domain decomposition of element-based grids. Either as preconditioners or solvers, domain decomposition methods have been used extensively in solving large sparse discretizations of partial differential equations [58] by separating the problem into easily parallelizable local interior problems and a separate Schur complement problem. When used as a preconditioning technique, so-called block-Jacobi methods solve the interior problems directly for use as a preconditioner to the full problem, often with sub-domain overlap to achieve condition numbers that scale as $C\left(1+(H / \delta)^{2}\right)$ where $H$ is the subdomain size, $\delta$ the overlap, and $C$ a constant independent of both [7, 47, 27, 25]. With the addition of a coarse-level correction, this bound can be reduced to $C(1+H / \delta)$ on quasi-regular grids. When used as a solver, domain decomposition techniques solve for the interface unknowns first using the Schur complement system, whose condition number scales as the inverse of the sub-domain size $h, C h^{-1}$, and can be significantly smaller in dimension than the full operator 8]. In doing so, the Schur problem is sometimes solved iteratively via multi-grid 4], conjugate gradients [16, 12, or GMRES 64, 37]. Coarse-grid corrected block-Jacobi preconditioners on the Schur problem reduce the condition number to $C\left(1+\log (H / h)^{2}\right)$ [12]. Thus, whenever possible, it is preferable to solve the Schur complement problem with adequate local and global coarse-grid preconditioning as opposed to solving a full Poisson system.

\subsection{The spectral multi-domain penalty method}

To discretize the two-dimensional Poisson-Neumann system a high-order discontinuous collocationbased variant of the spectral element method called the Spectral Multidomain Penalty Method (SMPM) [17, 21 is used. In the SMPM each element is assumed to be smoothly and invertibly mapped from a master element and the element connectivity is logically cartesian where each element has a single neighbor in 


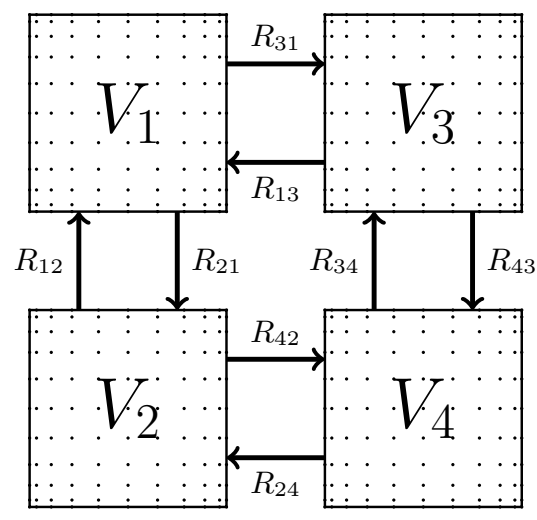

Figure 1: A depiction of the logical arrangement of a $2 \times 2$ element spectral multi-domain penalty method (SMPM) grid with $10 \times 10$ Gauss-Lobatto-Legendre points in each element denoted $V_{j}$ for $j=1,2,3,4$. The inter-element continuity fluxes are represented with $R_{i j}$ with $i \neq j$.

each of the north, south, east, and west directions. Within each element lies a two-dimensional GaussLobatto-Legendre (GLL) grid; denote as $n$ the number of GLL points per direction per element, and $m_{x}$ and $m_{z}$ the number of $x$ and $z$ elements in the grid 2 . On the GLL grid, a two-dimensional nodal Lagrange interpolant basis of polynomial order $n+1$ is constructed such that each basis element is one on one of the $n^{2}$ GLL points and zero on all of the others. This basis is used for approximating grid functions and their derivatives which are calculated by way of spectral differentiation matrices [14. The SMPM is a discontinuous method and so $C^{0} / C^{1}$ inter-element continuity and boundary conditions are only weakly enforced. While this method has been shown to be stable, spectrally accurate, and effective for under-resolved high-Reynolds number simulations of incompressible flows [17, 18, 1], the operator matrices resulting are unsymmetric, non-normal, and not positive definite. Lastly, due to the ill-posedness of the Poisson-Neumann problem, the operator matrix is rank-deficient, so the system must be made consistent prior to obtaining a solution. It is worth noting that although the SMPM matrices have several poor properties from a linear algebra perspective, the SMPM is chosen as the discretization here because of its demonstrated effectiveness for environmental flow problems, and also because its lack of a weak form makes its implementation relatively straightforward.

Now we define the SMPM element matrices and inter-element continuity conditions. In the subsequent discussion all operators (e.g. $\nabla^{2}$ ), element and domain boundaries (e.g. $\partial \Omega$ ) and domains are to be understood in the discrete sense; index notation is deprecated in favor of the continuous notation for convenience. For example, the domain $\Omega$ and the set of discrete points that is used to discretize $\Omega$ are used interchangeably when the intention is clear. All derivatives are computed by way of spectral differentiation matrices constructed from the Lagrange polynomial basis. Let $L u=f$ represent the discrete Poisson-Neumann system on $\Omega \subset \mathbb{R}^{2}$ a domain discretized into an $m_{x} \times m_{z}$ element mesh with each element $V_{i}$ smoothly and invertibly mapped from the master element $[-1,1] \times[-1,1]$. On each element a two-dimensional Gauss-Lobatto-Legendre (GLL) grid with $n$ points in each direction is constructed and used to evaluate the Lagrange interpolant basis and their derivatives by way of spectral differentiation matrices 14. Thus each element contains $n^{2}$ grid points. If $V_{i}$ and $V_{j}$ share the $n$ GLL points along one of their four boundaries, then each element owns a copy of those $n$ GLL nodes in order maintain the discontinuous nature of this method. Thus as a matrix, $L \in \mathbb{R}^{r \times r}$ is of dimension $r=n^{2} m_{x} m_{z}$, where $r$ denotes the total number of nodes in the grid $\Omega$.

In the SMPM the weak inter-element continuity condition is of Robin type, and is denoted by the flux $R_{i j}: \partial V_{j} \longrightarrow \partial V_{i}$ from element $V_{j}$ into $V_{i}$ for $V_{i}, V_{j}$ with an adjacent boundary $\partial V_{j} \cap \partial V_{i}$ consisting of $n$ grid points. $R_{i j}$ is defined as

$$
R_{i j}=I+\hat{n}_{i} \cdot \nabla
$$

where $\hat{n}_{i}: \partial V_{i} \longrightarrow \mathbb{R}^{2}$ is the outward pointing normal vector of $\partial V_{i}$ and $I$ is the identity operator. A depiction of a $2 \times 2$ element grid with the inter-element fluxes is shown in Fig. 1, in which the elements $V_{1}, V_{2}, V_{3}, V_{4}$ have been separated to emphasize the discontinuous nature of the SMPM.

\footnotetext{
${ }^{2}$ Here $z$ is the vertical direction as is convention in environmental fluid mechanics.
} 
The physical boundary conditions are Neumann, and are given on $\partial V_{i} \cap \partial \Omega$ as $n_{i} \cdot \nabla$ where $n_{i}$ is again the outward pointing normal vector. Given a function $u$, on an element $V_{i}$ the residual in the spectral multi domain penalty method is given by the sum of the Laplacian, the inter-element continuity mismatch, and the boundary condition mismatch as

$$
L_{i} u_{i}=\nabla^{2} u_{i}+\tau_{i}\left(R_{i i} u_{i}-\left.\sum_{j \in N(i)} R_{i j} u_{j}\right|_{\partial V_{i} \cap \partial V_{j}}\right)+\left.\tau_{i} \hat{n}_{i} \cdot \nabla u_{i}\right|_{\partial V_{i} \cap \partial \Omega}=f_{i}+\tau_{i} g_{i} .
$$

Here, $g_{i}$ is the boundary value of the Neumann boundary condition restricted to element $V_{i}$, and $N(i)$ is the index set of elements adjacent to $V_{i}$. The inter-element continuity, external boundary conditions, and the PDE are all satisfied weakly, since the residual is the sum of these three components. The penalty parameter $\tau_{i}$ represents the degree to which the inter-element continuity and boundary conditions are weighted in the residual relative to the PDE, and the optimal choice of $\tau_{i}$ is determined by stability criteria for hyperbolic problems [33, 34], and a heuristic for the Poisson problem [21].

\subsection{The Schur complement problem}

As shown in Figure 2 the domain $\Omega$ is discretized with a collection of elements $V_{j}$, each invertibly mapped from the master element $[-1,1] \times[-1,1]$. $\Omega$ is decomposed into $m_{x}$ many sub-domains $\Omega_{i}$, each a vertical strip of $m_{z}$ elements (see, for example, Fig. 2). Along each of the $m_{x}-1$ interfaces between the subdomains are $2 n m_{z}$ GLL nodes $\left(n m_{z}\right.$ nodes on either side of the interface). Denote as $k=2 n m_{z}\left(m_{x}-1\right)$ the number of interfacial nodes in the domain decomposition, and this set of $k$ interface nodes as $\Gamma$. The discrete Poisson operator $L$ (Eq. 4) is decomposed into a local term and an inter-subdomain flux term which is used to construct the Schur problem. This operator decomposition comprises three operators which are defined below.

First, denote as $E: \Gamma \longrightarrow \Omega$ the inclusion map that maps from the interfacial grid $\Gamma$ to $\Omega$. As a matrix, $E \in \mathbb{R}^{r \times k}$ and is composed of zeros and ones, $E^{T}$ is the restriction from the full grid to the interface grid $\Gamma$, and $E^{T} E=I \in \mathbb{R}^{k \times k}$ the identity matrix. Naturally $E E^{T}$ is not an identity matrix.

Second, define an operator $B: \Omega \longrightarrow \Gamma$ that consists of the inter-subdomain Robin boundary fluxes. $B$ represents all of the the inter-element fluxes $R_{i j}$ for $V_{i}$ and $V_{j}$ in different subdomains. As a matrix, $B \in \mathbb{R}^{k \times r}$, since it computes $I+\hat{n} \cdot \nabla$ within a subdomain using spectral differentiation matrices and assigns it to the interface of its neighbor.

Finally, define the operator $A: \Omega \longrightarrow \Omega$, which represents the part of $L$ that is entirely local to one subdomain. A consists of the Laplacian part of $L$, the boundary condition mismatch, all inter-element flux terms that are between elements contained in one subdomain, and the $R_{i i}$ terms in Eq. (4). Since $A$ is entirely local to each subdomain, as a matrix $A \in \mathbb{R}^{r \times r}$ is block-diagonal. $A$ represents $m_{x}$ decoupled homogenous Poisson-Robin boundary value problems, and as such is invertible and block diagonal.

These three operators are defined so that their combination yields the SMPM Poisson-Neumann operator

$$
L u=A u+E B u=f .
$$

Notice that the action of $E B$ couples the subdomains only weakly since its action is combined with $A$ in the residual $(A+E B) u=f$. This weak enforcement of inter-subdomain continuity in the SMPM allows for decoupling the subdomains by decoupling the action of $B$ from that of $A$. To accomplish this, we seek a vector $v \in \mathbb{R}^{k}$ on the interfacial nodes, $\bigcap_{i} \partial \Omega_{i}$, such that the solution to $A_{i} u_{i}=f_{i}-E v_{i}$ on each $\Omega_{i}$ subdomain also solves $L u=f$. By writing

$$
\mathrm{Au}=\mathrm{f}-\mathrm{EBu}
$$

it is clear that $v=B u$, the image of the solution under the inter-subdomain flux operator. Because $B$ is a contraction (mapping from the full grid to the interfacial grid), finding the image $B u$ is easier than finding $u$ itself; its value is given by the solution to the system

$$
\left[\begin{array}{rr}
A & E \\
B & -I
\end{array}\right]\left[\begin{array}{l}
u \\
v
\end{array}\right]=\left[\begin{array}{l}
f \\
0
\end{array}\right]
$$


As is evident, any $[u, v]^{T}$ that solves this system also solves $L u=f$, and satisfies $v=B u$; this system represents splitting the range of $A$ and $B$ in obtaining a solution of $L$. Taking one step of block Gaussian elimination of $A$ in this matrix to result in the upper triangular system

$$
\left[\begin{array}{rr}
I & A^{-1} E \\
0 & -I-B A^{-1} E
\end{array}\right]\left[\begin{array}{l}
u \\
v
\end{array}\right]=\left[\begin{array}{r}
A^{-1} f \\
-B A^{-1} f
\end{array}\right],
$$

we then obtain $v$ as the solution to the Schur complement system

of $A$ in Eq. (7) of

$$
\left(I+B A^{-1} E\right) v=B A^{-1} f
$$

A back-substitution of $v$ into Eq. (8),

$$
u=A^{-1}(f-E v)
$$

results in $u$ the solution of $L u=f$. Because the SMPM is a discontinuous element discretization, $A$ is block diagonal and invertible, and so all divisions of $A$ are easily parallelized; the expensive part of the above is obtaining the solution of the Schur complement system. In the rest of this paper, the focus is on efficiently obtaining this solution. To conclude, although this method of decomposing $L$ into its constituent components has a geometric interpretation in terms of decoupled homogenous boundary value problems, it is perhaps better understood as an algebraic method closely related to past novel uses of the Sherman-Morrison-Woodbury identity to update the inverse of a matrix with the addition of a low-rank modification [32. Algebraically, $A$ represents the easily-invertible matrix whose additive rank- $k$ modification is $E B$. Geometrically, $A$ represents decoupled Poisson boundary value problems and $E B$ the coupling between them. Plainly, both interpretations are valid and informative, and so we use the machinery of domain decomposition throughout to build a preconditioner for an iterative solver for Eq. (9), the algebraically obtained Schur complement system.

\subsection{Inconsistency of the Poisson-Neumann system}

Prior to obtaining the solution to Schur complement system, there remains the important point of dealing with the rank-deficiency of the Poisson-Neumann operator $L$. The Poisson-Neumann equation is ill-posed in the continuous sense, and so the SMPM operator $L$ is rank-deficient and has non-trivial left and right null spaces of dimension one. In symmetric discretizations, the kernel vector is the constant vector, but since $L$ is unsymmetric its left and right null spaces are different and only the right null space is constant vector. To ensure consistency and solvability the right-hand-side vector $f$ is projected out of the left null space of $L$ [50] and instead of $L u=f$, the regularized system solved is

$$
L u=\tilde{f}
$$

where $\tilde{f}=f-u_{L} u_{L}^{T} f$ is $f$ projected onto the range space of $L$ and $u_{L} \in \mathbb{R}^{r}$ is the unique vector with unit norm that satisfies $\left\|u_{L}^{T} L\right\|_{2}=0$. The solution $u$ then is only known up to an indeterminant additive constant vector. The rank deficiency of $L$ is inherited by the Schur complement system, and a relationship between the left null spaces of the Schur complement system and full Poisson matrix is shown in the following claim.

Claim 1. Denote as $u_{L} \in \mathbb{R}^{r}$ the left null vector of $L \in \mathbb{R}^{r \times r}$. Denote as $u_{S} \in \mathbb{R}^{k}$ the left null vector of $S \in \mathbb{R}^{k \times k}$. Then the following relations hold.

1. $u_{S}=E^{T} u_{L}$

2. $u_{L}=A^{-T} B^{T} u_{S}$

Proof. See appendix. 
The rank-deficiency of the Schur complement matrix means another regularization is required to project the Schur right hand side $b_{S}=B A^{-1} \tilde{f}$ out of the left null space of the Schur complement system. Thus the Schur complement system,

$$
S x_{S}=b_{S}
$$

is modified to read

$$
S x_{S}=b_{S}-u_{S} u_{S}^{T} b_{S}
$$

To summarize, the method for obtaining the solution $u$ to $L u=f$ is shown in Algorithm 1 . The statement $\operatorname{SOLVE}\left(S, b_{S}\right)$ in Step 4 is meant to represent the solution of the linear system $S x_{S}=b_{S}$.

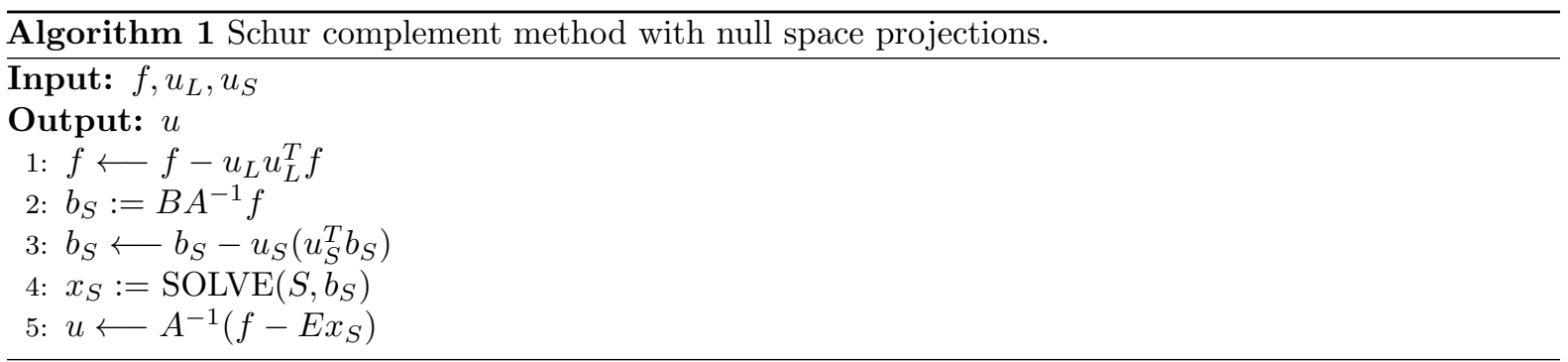

The second regularization, Step 3 , is done to ensure that the Schur complement system is consistent, and thus, solvable in Step 4. However, it is not obvious that modifying the right hand side of the Schur complement system is acceptable from the perspective of solving the Poisson-Neumann equation. How do we know that modifying the right hand side of the Schur complement system does not give us the wrong answer $x_{S}$ for Step 5? Fortunately, it can be shown that so long as the original Poisson-Neumann system was made consistent (e.g. Eq. (11) ) the regularization of the Schur problem does not affect the accuracy of the solution of the Poisson equation. This is shown in the following claim.

Claim 2. Given $L u=\tilde{f} \in \mathbb{R}^{r}$, if $u_{L}^{T} \tilde{f}=0$, then the error in the solution $u=A^{-1}\left(\tilde{f}-E x_{S}\right)$ recovered from the solution of the regularized Schur complement system $S x_{S}=b_{S}-u_{S} u_{S}^{T} b_{S}$ is bounded by the error of Schur complement solution:

$$
\|L u-\tilde{f}\|_{2} \leq\left\|S x_{S}-\left(I-u_{S} u_{S}^{T}\right) b_{S}\right\|_{2}
$$

Proof. See appendix.

Finally, a means to obtain $u_{L}$ and $u_{S}$ is required. Note that first $\left\|u_{S}^{T} S\right\|_{2}=0$ means that $u_{S}$ is a left eigenvector of $S$. Thus, shifted inverse iteration is used on $S^{T}-\sigma I$ to find the left kernel vector $u_{S}$, and then this vector is used to reconstruct the left kernel vector $u_{L}$ by way of the second relation in Claim 1 . With a small shift of $\sigma$ and a zero eigenvalue $\lambda_{n}=0$, the convergence of shifted inverse iteration is of order $\left(\sigma-\lambda_{n}\right)^{-1}=\sigma^{-1}$. Since $\sigma$ is usually chosen to be close to zero, this iteration converges quickly, often in one or two steps.

\section{Solving the Schur complement system}

\subsection{A scalable preconditioning algorithm}

Many preconditioning techniques for the Schur complement system have been proposed [64, 16, 12, 65] with an aim towards algorithmic scalability. Algorithmic scalability refers to the property that an iterative solution method converges independently of the number of degrees of freedom of the underlying grid; in this case it means that the number of Krylov method iterations is bounded as $m_{x}$ grows, but in general it is understood to mean that the conditioning of the preconditioned matrix is independent of a grid parameter of interest. For example, scalability is sometimes defined to mean that the conditioning of a system does not deteriorate as the number of subdomains grows (see Definition 1.3 in Toselli and Widlund [58]) or for high-order methods that the conditioning does not worsen as the polynomial degree is increased (see Definition 1.2 in [58] or Section 4 in [27]). 
To achieve algorithmic scalability, most preconditioning techniques for elliptic problems rely on two preconditioners, a local preconditioner that can be applied in parallel and a coarse global preconditioner to speed across-grid communication of components of the residual. An example is the two-level additiveSchwarz preconditioner in which overlapping block-diagonal components are solved in parallel, augmented with a coarse grid correction to communicate information across the grid [26, 21]). In this paper, a non-overlapping block-diagonal/block-Jacobi preconditioner is used, augmented with deflation, to achieve Krylov subspace convergence rates independent of $m_{x}$. As $m_{z} \ll m_{x}$ in long domain problems, achieving convergence independent of $m_{x}$ is of crucial importance.

It is important to distinguish algorithmic scalability from parallel scalability which refers to the property that the time taken to execute an algorithm decays to zero as the number of CPU cores is increased (strong scaling), or that work can be done in constant time if the work per CPU core is kept constant (weak scaling). Achieving parallel scalability requires both a scalable algorithm as well as an efficient parallel implementation of that algorithm. Furthermore, the implementation of Krylov solvers requires great care to avoid parallel bottlenecks at scale, and recent research efforts have been targeted at pipelining portions of the Krylov subspace algorithm to avoid latency [30, 36]. These details of implementation are important, but, tangential to the development of a preconditioning algorithm. Therefore, in this paper we only discuss algorithmic scalability as we focus on creating a preconditioning algorithm that is scalable with respect to grid parameters of interest.

\subsection{Krylov solver implementation}

The iterative solution method used in Step 4 of Algorithm 1 and throughout this work is the Generalized Minimum Residual Method (GMRES) [51. GMRES was chosen because of its generality as it is capable of solving unsymmetric linear systems like the ones in SMPM, and requires only the ability to compute matrix-vector products of the operator matrix $S$. Further, even though the Schur complement matrix inherits singularity from the Poisson matrix, when made consistent the Schur system converges properly in GMRES. This is first because the left null and right range spaces of $S$ are orthogonal, a necessary condition for convergence of GMRES [10] on singular matrices, and second because every principle sub-matrix of $S$ is invertible. Thus, after projection out of the null space, rank deficiency only plays a role in the final Krylov space which is of course never reached in practice. However, the non-normality of $S\left(S S^{T} \neq S^{T} S\right)$ means that the conditioning and more generally the spectrum alone is not predictive of GMRES convergence 31, 44. For the remainder of the discussion, then, iteration counts will be used as the metric for evaluating the performance of the various preconditioning techniques.

In this implementation, the blocks of the Schur matrix, denoted $S_{i}$ for an interface $\Gamma_{i}$ between subdomains $\Omega_{i}$ and $\Omega_{i+1}$, are assembled, stored, and multiplied against vectors in a parallel sparse distributed fashion. Each such block is of dimension $S_{i} \in \mathbb{R}^{4 n m_{z} \times 2 n m_{z}}$. Assembling the Schur matrix takes advantage of the fact that the interfaces are sparse in the full grid for a high-order method and thus assembly and computation of matrix-vector products $\sum_{i} S_{i} x_{i}$ requires less work than the explicit computation of $\left(I+B A^{-1} E\right) x$. So long as storage of the all the blocks $\left\{S_{i}\right\}_{i=1}^{m_{x}-1}$ is possible, and the Poisson-Neumann problem is to be solved many times, it is preferable to assemble $S$.

Finally, note that the implementation of GMRES used here uses Householder reflections for orthogonalization in the Arnoldi process, which requires about three times more operations than the classic modified Gram-Schmidt (MGS) method. This choice is made because of a loss of orthogonality in the Krylov basis $V_{k}$ due to round-off error in MGS that scales with condition number [6],

$$
V_{k}^{T} V_{k}=I+\mathcal{O}\left(\epsilon \kappa_{2}(S)\right),
$$

where $\epsilon$ is machine precision and $\kappa_{2}(S)$ is the condition number of $S$. This is clearly problematic here as $S$ is singular. Walker 62] shows that by contrast, orthogonalization with Householder transformations yields an orthogonality error of

$$
V_{k}^{T} V_{k}=I+\mathcal{O}(\epsilon)
$$

which is a benefit that is worth the extra floating point operations.

\subsection{Non-overlapping block-Jacobi preconditioner}

For sparse matrices with non-zeros clustered around the diagonal, computing the inverse of blocks along the diagonal separately can be a useful preconditioning technique. Block-Jacobi preconditioners have been shown to be effective for the Schur complement of elliptic operators [15, especially when combined 
with coarsened-grid preconditioners 43, 49, 48. In the context of solving the Poisson equation, $A^{-1}$ represents a block-Jacobi preconditioner since $A$ represents decoupled local problems, each a Poisson-Robin boundary value problem. For preconditioning the Schur matrix $S$, a block-Jacobi preconditioner can also be assembled in which a single block represents the coupling between interfaces $\Gamma_{i}$ and $\Gamma_{i+1}$ in the Schur grid $\Gamma$. Each such block represents the coupling between four interfaces: the two bounding a subdomain $\Omega_{i}$ on the left and the right, and their adjacent interfaces in $\Omega_{i-1}$ and $\Omega_{i+1}$. This is pictorially depicted in Fig. 2, in which every four consecutive interfaces belonging to one block in the block-diagonal preconditioner are shown in the same color (either red or green), and represent the nodes belonging to $\Gamma_{i}$ and $\Gamma_{i+1}$.

To graphically show the relationship between the grid and the blocks in the block-Jacobi preconditioner, the sparsity pattern of the Schur matrix corresponding to the grid in Fig. 2 is depicted in black in Fig. 3 . The dimensions of the grid are $n=5, m_{z}=10, m_{x}=17$. Overlaid on the sparsity pattern of $S$ in Fig. 3 are the blocks of the block-Jacobi preconditioner used here, shown in alternating colors of green and red that correspond respectively to the green and red interfaces they represent in Fig. 2. Since each interface consists of $n m_{z}$ grid points, the dimension of each square block is $4 n m_{z}$, except for possibly the last such block which may be smaller if $m_{x}$ is odd. Denoting the block-Jacobi preconditioner matrix as $M$, the preconditioned Schur system that is solved with GMRES is

$$
S M^{-1} x_{S}^{\prime}=b_{S}
$$

and the solution is obtained by a final division by $M$

$$
x_{S}=M^{-1} x_{S}^{\prime} .
$$

Since $M$ is explicitly block-diagonal (i.e. any non-zeros of $S$ coupling the blocks of $M$ are ignored in the factorization of $M$ ), divisions by $M$ can be computed efficiently in parallel. Finally note that although the preconditioner is algebraically non-overlapping, an application of the preconditioner connects grid points from subdomain $\Omega_{i-1}$ to $\Omega_{i+1}$, which is similar in practice to an overlapping preconditioner on the full Poisson operator $L$.

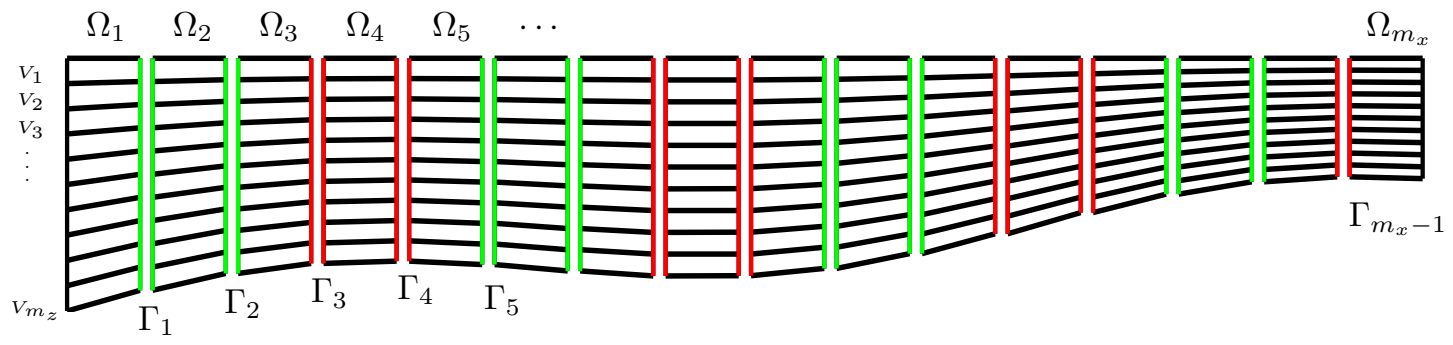

Figure 2: A sample domain with $m_{x}=17$ and $m_{z}=10$ elements in $x$ and $z$ respectively. Each vertical strip of 10 elements is a sub-domain $\Omega_{j}$, and the collection of interfaces $\left\{\Gamma_{k}\right\}_{k=1}^{m_{x}-1}$ represents the grid for the Schur complement problem. The red/green interfaces correspond to the red/green blocks in the sparsity pattern of the block-Jacobi preconditioner shown in Fig. 3

\subsection{Deflation}

Working in tandem with other preconditioners, deflation methods aim to accelerate the convergence of Krylov methods by eliminating (or "deflating") components of the residual within a chosen subspace. The subspace is usually chosen to be a span of approximate eigenvectors of the operator corresponding to slowly converging eigenvalues. Thus, the problematic eigenvalues are solved directly using a coarsened version of the operator, and the remaining components of the residual are eliminated by a Krylov solver. The first deflation methods were used to accelerate the convergence of the conjugate gradient method for elliptic boundary value problems [46, and thus were limited to symmetric positive definite matrices. The extension to unsymmetric matrices [20] and in particular applications related to domain decomposition with preconditioning 61 and fluid flow 55 make deflation a good candidate for augmenting block-Jacobi preconditioning in our Schur problem. Comparisons of coarse grid correction with deflation show that deflation methods combined with preconditioning yield lower condition numbers irrespective of the choice 


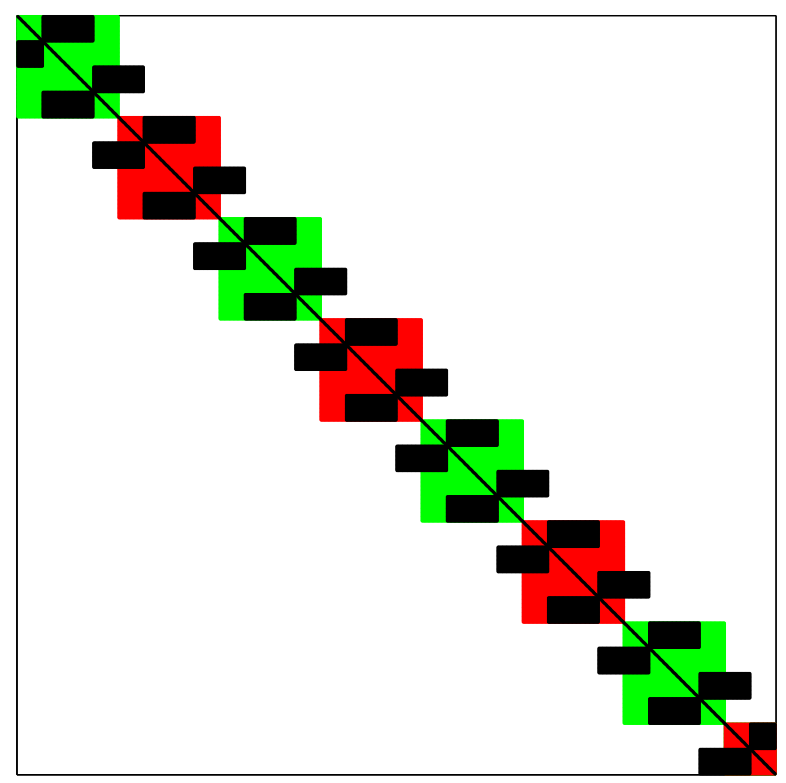

Figure 3: The Schur matrix shown in black corresponding to a grid with $n=5, m_{x}=17$, and $m_{z}=10$ shown in Fig. 2 . with the blocks $M_{i}$ of the non-overlapping block-Jacobi preconditioner $M$ in red and green. Each block-Jacobi block represents the coupling between four of the interfaces shown in Fig. 2 in which the red/green interfaces correspond to the red/green blocks shown here.

of the coarsening operators for symmetric positive-definite systems [4], and deflation methods have been elsewhere experimentally favorably compared in the context of two-level preconditioners with multi-grid and domain decomposition approaches [57, 56]. A more exhaustive summary and analysis of deflation methods can be found in Ref. 28].

Here, to augment the block-Jacobi preconditioner described in Section 3.3 , a deflation method is used as the coarse-grid correction method, following the procedure in Ref. 20. The deflation vectors are chosen to be a set of $d$ column vectors $Z \in \mathbb{R}^{k \times d}$ where $d \ll k$, and $k=\operatorname{dim}(S)$. These deflation vectors are chosen to be discrete indicator vectors on each of the interfaces $\left\{\Gamma_{j}\right\}_{j=1}^{m_{x}-1}$ that form the Schur grid $\Gamma$. The $i$-th entry in the $j$-th such vector is given by

$$
\left(z_{j}\right)_{i}=\left\{\begin{array}{ll}
1 & : \text { if } x_{i} \in \Gamma_{j} \\
0 & : \text { if } x_{i} \notin \Gamma_{j}
\end{array}\right\}
$$

thus each vector is active on one interface in the Schur grid. The matrix of these vectors $Z=\left[z_{1}, z_{2}, \cdots, z_{d}\right]$ defines a coarse version of the Schur problem, $C=Z^{T} S Z \in \mathbb{R}^{d \times d}$, and two projections

$$
\begin{aligned}
& P=I-S Z C^{-1} Z^{T} \\
& Q=I-Z C^{-1} Z^{T} S
\end{aligned}
$$

each of size $\mathbb{R}^{k \times k}$. The operator $Z$ defines a one-dimensional coarse grid of dimension $d$ that is composed of the mean $x$-coordinates of each of the $m_{x}-1$ interfaces $\Gamma_{j}$. As a matrix $Z^{T} \in \mathbb{R}^{d \times k}$ is a contraction operator that maps grid functions on the Schur grid to the coarse grid. The mapping $y=Z^{T} x$ sums up the values of $x$ along each interface $\Gamma_{j}$ and stores them in $y_{j}$. The mapping $x=Z y$ is the prolongation operator which assigns to all entries of $x$ on $\Gamma_{j}$ the value $y_{j}$. Note that $Z^{T} Z=n m_{z} I \in \mathbb{R}^{m_{x}-1} . Z Z^{T} x$ averages the values of $x$ over each interface $\Gamma_{j}$. The intuition behind the projections $P$ and $Q$ is that they project out of the subspace on which $Z C^{-1} Z^{T}$ is a good approximation of the left (in the case of $Q$ ) or right (in the case of $P$ ) inverse of $S$. Thus the projections map onto the complement of the subspace on which the coarse matrix $C$ approximates the Schur matrix $S$ well.

Departing from the method in Ref. 20, the division by $C$ is regularized to address $C$ 's rank-deficiency inherited from $S$. This regularization is identical to the null-space projection described in Section 2.4 with the kernel basis vector of $C$ denoted $u_{C}$ and satisfying $\left\|u_{C}^{T} C\right\|=0$. The projections $P$ and $Q$ are also regularized in the same way. Note that the left null space of $C$ is one dimensional, and is spanned by 
$Z^{T} u_{S}$. Denote this vector $u_{C}=Z^{T} u_{S}$ and write the regularized projections as

$$
\begin{aligned}
& P=I-S Z\left(C \backslash\left(Z^{T}-u_{C} u_{C}^{T} Z^{T}\right)\right) \\
& Q=I-Z\left(C \backslash\left(Z^{T}-u_{C} u_{C}^{T} Z^{T}\right)\right) S .
\end{aligned}
$$

Deflation proceeds by noting that the solution of the Schur complement problem $S x_{S}=b_{S}$ can be decomposed into

$$
x_{S}=(I-Q) x_{S}+Q x_{S} .
$$

Then, the first term is just $Z C \backslash\left(Z^{T}-u_{C} u_{C}^{T} Z^{T}\right)\left(b_{S}-u_{S} u_{S}^{T} b_{S}\right)$, which can be computed directly since $C$ is small. The second term is obtained by performing GMRES on the deflated and right-preconditioned system $P S M^{-1} x_{S}=P\left(b_{S}-u_{S} u_{S}^{T} b_{S}\right)$ and then post-multiplying by $Q$, finally assembling the solution as

$$
\begin{aligned}
x_{S} & =Z C \backslash\left(Z^{T}-u_{C} u_{C}^{T} Z^{T}\right)\left(b_{S}-u_{S} u_{S}^{T} b_{S}\right) \\
& +Q M^{-1} \operatorname{GMRES}\left(P S M^{-1}, P\left(b_{S}-u_{S} u_{S}^{T} b_{S}\right)\right) .
\end{aligned}
$$

Because $P$ projects out of the coarse space, the GMRES solution of $P S M^{-1} x_{S}=P\left(b_{S}-u_{S} u_{S}^{T} b_{S}\right)$ minimizes only the component of the residual that cannot be well-approximated by the coarse solution. Note that although the deflated operator $P S M^{-1}$ is rank-deficient by virtue of the rank-reducing projection $P$, because the the right-hand-side vector is similarly projected the GMRES algorithm converges properly. This formulation of deflation-augmented right-preconditioning is an extension of the work in Ref. [20] to a rank-deficient matrix.

With reference to implementation, the multiplication of matrices $Z^{T}$ and $Z$ into vectors is very easily parallelizable since $Z^{T} x$ is a global reduction easily implemented in MPI and $Z x$ is a local extension from the coarse grid to the Schur grid that requires no communication. Furthermore since $P$ and $Q$ are never explicitly formed, the storage overhead for deflation is merely the storage of the coarse matrix $C$ which is a one-dimensional tridiagonal finite-difference Poisson matrix of dimension $m_{x}-1$ whose solution is obtained by the Thomas algorithm. The applications of $P, S$, and $M^{-1}$ required in each iteration of GMRES are conducted in a distributed, sparse, and MPI-parallel fashion, with the exception of the coarse solution, which requires global communication between all MPI ranks.

For completeness, Algorithm 2 depicts the algorithmic summary of the deflation method in which the notation $\operatorname{GMRES}(A, b)$ is intended to represent the solution of a linear system $A x=b$ with GMRES. The algorithm expects to be given the vectors spanning the left null spaces of $C, S$, and $L\left(u_{C}, u_{S}, u_{L}\right.$ respectively) and returns the solution $x$ that makes $L x-b$ small. Naturally there are input parameters for GMRES that are omitted in the description below.

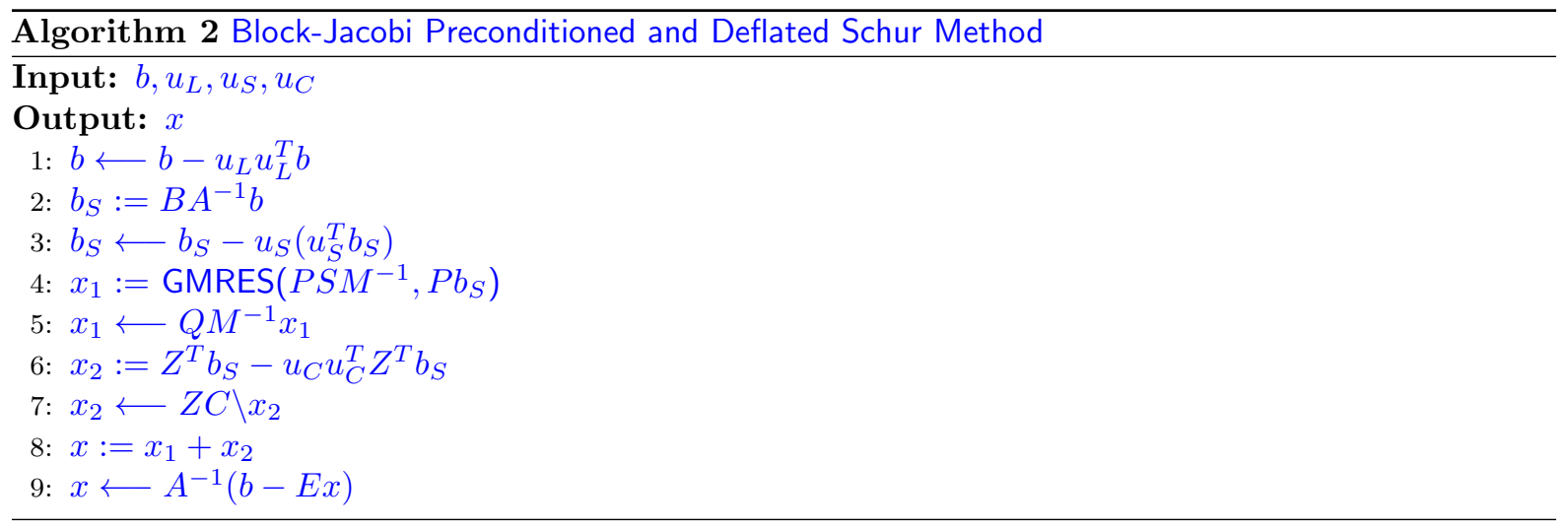

\subsection{Two-level block-Jacobi preconditioner}

Finally, note that the two-level block-Jacobi method can also be written using the deflation vectors $Z$ defined above as coarsening operations. Denoting the coarse problem again as $C=Z^{T} S Z$, write the two-level block-Jacobi preconditioned system as

$$
S\left(M^{-1}+Z C \backslash\left(Z^{T}-u_{C} u_{C}^{T} Z^{T}\right)\right) x_{S}=b_{S}-u_{S} u_{S}^{T} b_{S} .
$$


As defined above, the two-level block-Jacobi preconditioner will be used to compare against the performance of the deflation method. Notice that although they appear similar, the block-Jacobi method only requires one application of the Schur matrix $S$ per Krylov iteration whereas the deflation method requires two, with a second embedded in the definition of $P$. Furthermore, unlike the deflation method does with its projection $P$, block-Jacobi preconditioning doesn't explicitly project out of the coarse space. Thus while the Krylov solver is aided by the coarse matrix $C$, the residual minimized still contains components in the coarse space. Finally notice that right-preconditioning has been used throughout. This is so that the the tolerance achieved on the preconditioned system bounds the tolerance on the unpreconditioned system, and not the other way around as when left preconditioning is used. The algorithmic description of the two-level block-Jacobi method is given in Algorithm 3 .

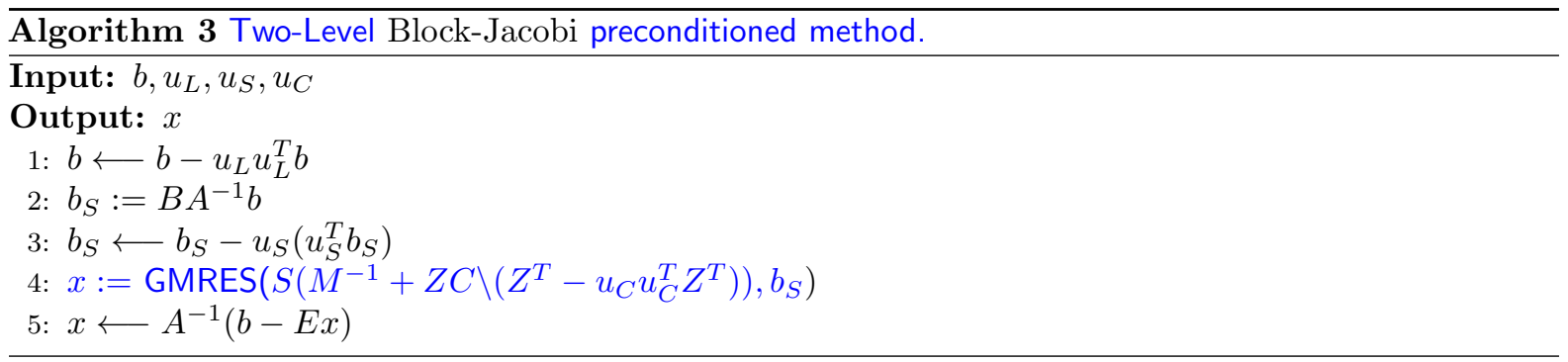

\section{Performance}

In this section a performance comparison of four different preconditioning methods for solving the Schur complement problem using GMRES is made. The four methods are

1. no preconditioning

2. block-Jacobi preconditioning

3. deflation-augmented block-Jacobi preconditioning

4. two-level block-Jacobi preconditioning

These are all described in Section 3, and are summarized in Table 1. The basis for comparison will be the number of iterations to achieve a GMRES relative tolerance of $10^{-10}$, as well as the wall-clock time taken to achieve that tolerance. Both iteration count and computation time are relevant since each of the four methods has a different per-Krylov iteration computational cost. In all cases, the right hand side used is a randomly generated vector drawn from a uniform distribution on [0,1], and the measurement of iteration count and computation time is averaged over the solution of ten such right hand sides. The initial guess is always the vector of zeros, and the solution $x_{S}$ is always checked to verify that

$$
\frac{\left\|S x_{S}-b_{S}\right\|_{2}}{\left\|b_{S}\right\|_{2}}<10^{-10} .
$$

Two classes of problems are studied to show GMRES convergence independent of grid properties to asses algorithmic scalability (c.f. Section 3.1). First, to show that the block-Jacobi preconditioner alone eliminates the dependence on domain aspect ratio, the Poisson problem is solved on a series of increasingly stretched grids. Second, to show that the projections in the deflation method eliminate the dependence on $m_{x}$, a series of grids with increasingly many $x$ elements is constructed on which the Poisson equation is solved. In the second set of problems, deflation is compared with two-level block-Jacobi preconditioning to demonstrate that deflation requires both fewer GMRES iterations and less wall-clock time. These computations are performed in an MPI-parallel Fortran code and are executed on a four processor AMD Opteron 6366HE computer with 64 cores using version 5.3 of the AMD Core Math Library for all basic linear algebra tasks, and version 3.1 .3 of the MPICH2 MPI library. 


\section{Name}

No Preconditioning

Block-Jacobi

Deflation and Block-Jacobi

Two-Level Block-Jacobi

Abbreviation
Schur
BJ-Schur
DBJ-Schur
TLBJ-Schur

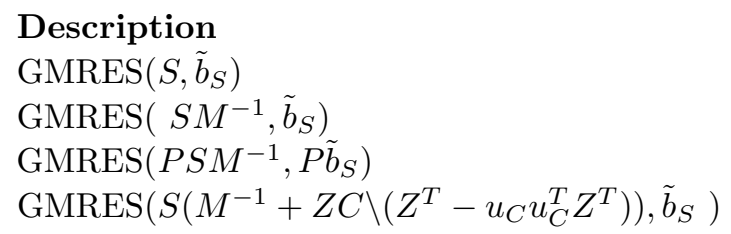

Table 1: A description of the four methods compared in this section. $\operatorname{GMRES}(A, b)$ represents a GMRES solution of the linear system $A x=b$.

\subsection{Increasing domain aspect ratio}

The goal of this section is to demonstrate that use of the block-Jacobi preconditioner on the Schur matrix eliminates the dependence of GMRES convergence on the domain aspect ratio. To show this, the Poisson-Neumann problem was solved on a series of domains with constant height of $l_{z}=10 \mathrm{~m}$ and incrementally increasing width from $l_{x}=10 \mathrm{~m}$ to $l_{x}=5000 \mathrm{~m}$. These domains are discretized with 10 elements in both the horizontal and the vertical, yielding an element aspect ratio $\eta=\left(l_{x} / m_{x}\right) /\left(l_{z} / m_{z}\right)$ that grows from $\eta=1$ to $\eta=500$.

On this set of grids the Schur problem is assembled and solved with and without block-Jacobi preconditioning. Shown in Fig. 6(b) are the residual histories of the unpreconditioned GMRES solver for five such grids with aspect ratios increasing from $\eta=1$ to $\eta=500$. Notice that as the aspect ratio increases, there is an increasingly long period of slow decay of the GMRES residual before it quickly achieves the desired tolerance. As $\eta$ increases to 500, the number of GMRES iterations grows from under about 80 to 1500, a twenty-fold increase that is expected due to the ill-conditioning of the spectral differentiation matrices that worsens as the element aspect ratio grows 35 .

As shown in Fig. 4(a), and in contrast to the unpreconditioned Schur method, the same set of problems solved with block-Jacobi preconditioning shows no dependence on aspect ratio, converging to the tolerance in roughly 20 GMRES iterations for all values of $\eta$. Notice though that the iteration count in the unpreconditioned Schur method does not grow indefinitely; it plateaus after $\eta>150$ at its final value of $\sim 1000$. This behavior can be explained by understanding that for $\eta>150$ all horizontal wavenumbers supported by the grid are longer than the supported vertical wavenumbers. As such, the discrete eigenvalue spectrum of the Poisson operator is separated into two distinct sets, a condition that defines leptic grids. This separation of eigenvalues presents a difficulty for Krylov subspace methods as has been demonstrated previously 53, but once the horizontal and vertical eigenvalues are separated entirely further distortion of the grid only minimally affects the convergence properties of GMRES. Finally, both methods show a peak in iterations near $\eta=75$, a result that is not well understood.

Commensurate with the difference in iteration count, block-Jacobi preconditioned Schur is significantly faster than the unpreconditioned Schur method. The wall-clock computation time for both methods is shown in Fig. 4(b), which demonstrates that with the use of the block-Jacobi preconditioner a solution is obtained in $\mathcal{O}\left(10^{-1}\right)$ seconds for all aspect ratios. By contrast, the time to solution for the unpreconditioned Schur method grows from $\mathcal{O}\left(10^{-1}\right)$ to $\mathcal{O}\left(10^{1}\right)$ seconds as the aspect ratio increases. Lastly note that the results for the deflated and two-level block-Jacobi solvers are not shown, since for a small $10 \times 10$ element grid the coarse-grid correction does not improve performance significantly.

\subsection{Increasing the number of $x$ elements}

Next is the study of the performance of the various GMRES preconditioning methods as the number of $x$ elements is increased. This study gives an insight into how the four methods will perform on environmental flow problems in which not only is the domain aspect ratio large, but the number of elements in the long direction is also large. In this study, $n=10$ and $m_{z}=10$ were fixed, and the Poisson-Neumann problem is solved for discretizations with $m_{x}=64$ to $m_{x}=1024$ on domains with increasing length $l_{x}$. The number of grid points grows from 64000 , when $m_{x}=64$, to 1024000 , when $m_{x}=1024$. For each such grid, the Schur problem was assembled and solved with the four preconditioning techniques described at the beginning of this section. Each computation was performed ten times and the iteration counts and timings were averaged over these 10 trials. Fig. 5(a) shows the resulting number of iterations required to achieve a tolerance of $10^{-10}$ for each of the four solvers as a function of the number of $x$ subdomains $m_{x} \in[64,1024]$. Notice that the deflated block-Jacobi preconditioner achieves GMRES convergence independent of $m_{x}$ and converges within approximately 30 iterations, achieving a bound on 
(a)

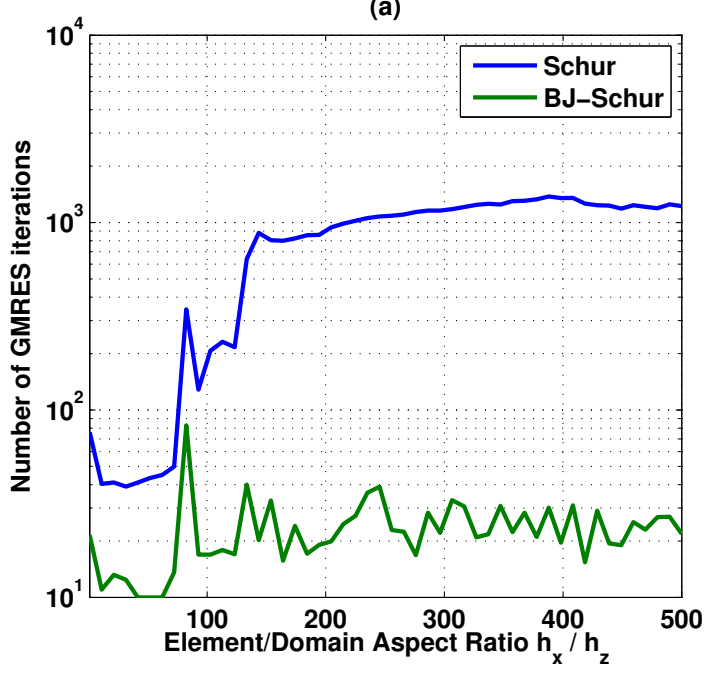

(b)

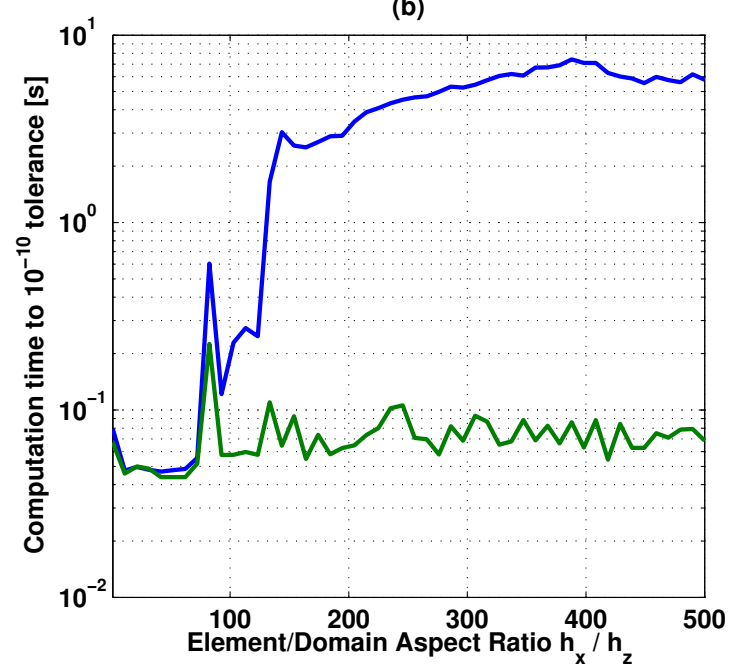

Figure 4: Left: Number of GMRES iterations to solution as a function of element and domain aspect ratio for a $10 \times 10$ element grid with 100 points per element. Right: Time to solution the same problem as a function of element and domain aspect ratio. This was benchmarked with an MPI-parallel Fortran code on 5 MPI ranks.

the number of iterations with increasing $m_{x}$ that is vital for good performance in applications with long domains. The two-level block-Jacobi method also shows convergence independent of problem size but takes twice as many GMRES iterations as deflation in all cases.

Since the cost of $K$-many GMRES iterations is $\mathcal{O}\left(K^{3}\right)$, and iterations grow in the unpreconditioned Schur method linearly with $m_{x}$, it is expected that the any coarse-grid corrected method cubically outperforms the unpreconditioned Schur method in solution time as $m_{x}$ becomes large. In practice, the speedup is closer to quadratic than cubic in $m_{x}$ because the solution of the coarse problem requires far more communication than the Schur method alone.

A comparison of the four methods' time-to-solution is presented in Fig. 5(b), which shows that the speedup in deflation over block-Jacobi preconditioned Schur is $\mathcal{O}\left(m_{x}^{2}\right)$. The two-level block-Jacobi method shows similar quadratic in $m_{x}$ speedup relative to the Schur method, but is always slower than the deflation method since it takes about twice as many GMRES iterations to converge. The difference in time between the deflated and block-Jacobi methods is smaller than the factor of two difference in GMRES iterations because each GMRES iteration of the deflation method requires two applications of the $S$ matrix due to the additional $S$ application in the $P$ projection; the two-level block-Jacobi method requires only one $S$ matrix-vector multiply. Nevertheless, deflation augmented preconditioning outperforms two-level block-Jacobi preconditioning in terms of wall-clock time for all of the grids studied here by about $25 \%$.

Fig 6(a) shows a representative example of the residual history for the case where $m_{x}=512$. The residual histories of both the block-Jacobi and unpreconditioned cases show an initial period of slow convergence followed by a rapid decay in the residual. This slow convergence is associated with the first few singular values which represent low-frequency components within the Schur matrix whose elimination requires across-grid communication. This initial slow convergence behavior is eliminated by either the coarse-grid solve in two-level block-Jacobi or by the deflation vectors in the deflation preconditioner. However, it is clear that the deflation method yields better GMRES performance since the residual decays at a rate twice that of the two-level block-Jacobi residual.

Although both deflation and block-Jacobi methods demonstrate algorithmic scalability in $m_{x}$, it is always preferable to use the deflated Schur method over the two-level block-Jacobi method, both from the perspective of minimizing storage (in the number of Krylov basis vectors required) as well as minimizing computation time. The deflation method takes about half as many iterations, and about $25 \%$ less time. The results shown in Fig. 5 are quantified in Table 2, along with data showing the amount of setup time required to assemble the Schur complement matrix, its coarse version, and factor its preconditioner. Setup takes about 100 times longer than a single solve, meaning that its cost is easily amortized over the $\mathcal{O}\left(10^{4}-10^{5}\right)$ solves required in a time-evolving incompressible Navier-Stokes simulation. 
(a) Iterations

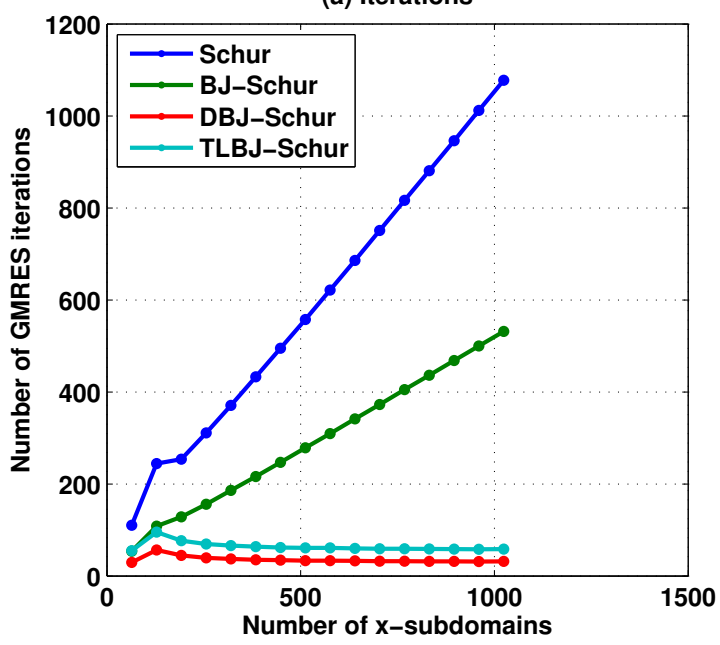

(b) Computation time

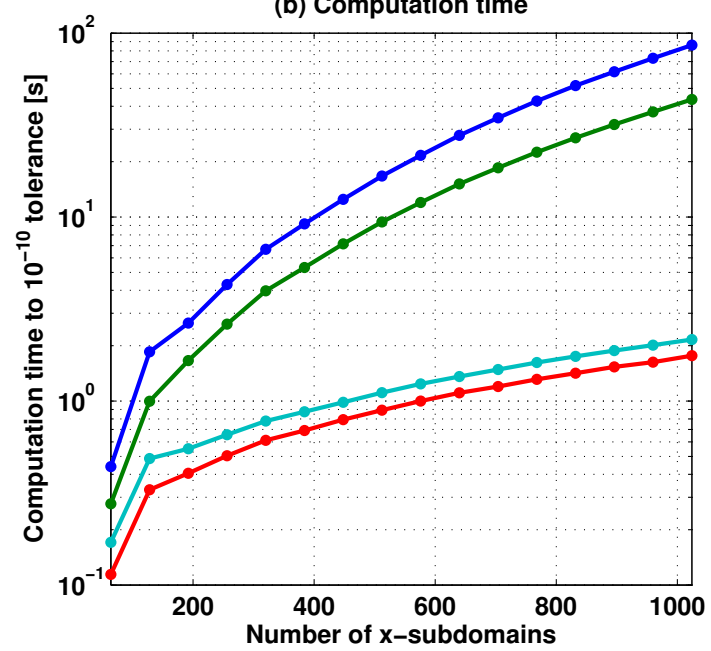

Figure 5: Left: Number of GMRES iterations to solution for a constant aspect ratio as a function of the number of $x$ subdomains. Right: Time to solution for a constant aspect ratio as a function of the number of $x$ subdomains. This simulation was benchmarked with $\left(n, m_{z}\right)=(10,10)$ fixed, and a GMRES tolerance of $10^{-10}$ on $16 \mathrm{MPI}$ ranks on an Opteron $6366 \mathrm{HE}$ architecture.

(a)

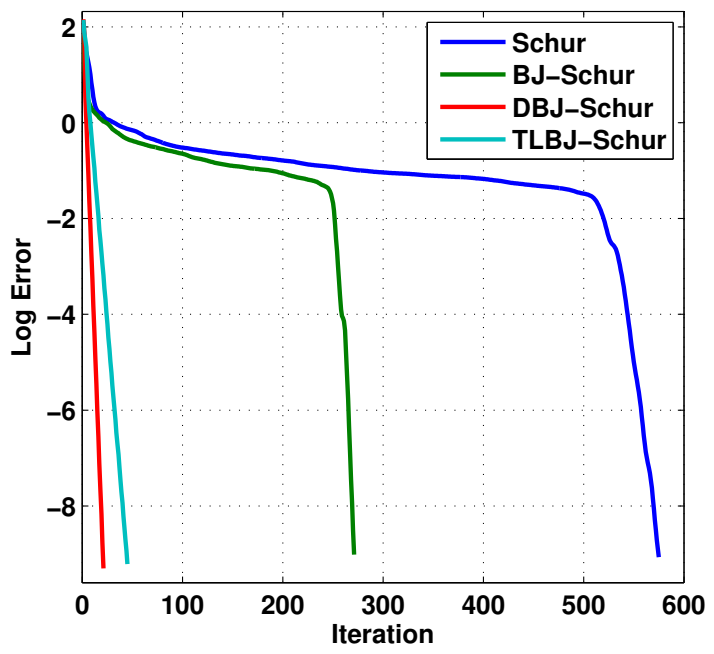

(b)

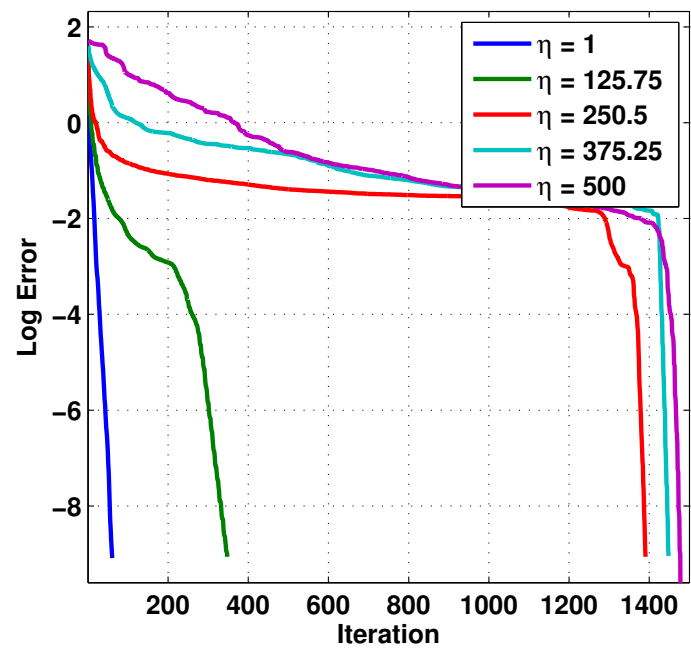

Figure 6: Left: The GMRES relative error as a function of iteration for a $10 \times 512$ element grid with $n=10$ for each of the four methods compared. Right: The GMRES relative error as a function of iteration for five different aspect ratios $\left(\eta=h_{x} / h_{z}\right)$ with the unpreconditioned Schur method on a $10 \times 10$ element grid with $n=10$.

\begin{tabular}{|c|c|c|c|c|c|c|c|c|c|c|}
\hline \multirow[b]{2}{*}{$m_{x}$} & \multirow[b]{2}{*}{ Grid Points } & \multirow[b]{2}{*}{ Setup Time } & \multicolumn{2}{|c|}{ Schur } & \multicolumn{2}{|c|}{ Block Jacobi } & \multicolumn{2}{|c|}{ Deflated block-Jacobi } & \multicolumn{2}{|c|}{ TL block Jacobi } \\
\hline & & & Iter. & Time & Iter. & Time & Iter. & Time & Iter. & Time \\
\hline 64 & $6.4 \times 10^{4}$ & 42.4 & 110.5 & 0.44 & 54.1 & 0.276 & 29.6 & 0.114 & 54.9 & 0.171 \\
\hline 128 & $1.28 \times 10^{5}$ & 84.9 & 244.4 & 1.85 & 108.6 & 0.997 & 56.7 & 0.329 & 95.4 & 0.487 \\
\hline 256 & $2.56 \times 10^{5}$ & 168.0 & 311.1 & 4.30 & 156.2 & 2.61 & 39.3 & 0.504 & 69.7 & 0.656 \\
\hline 512 & $5.12 \times 10^{5}$ & 327.0 & 557.7 & 16.7 & 278.6 & 9.40 & 33.4 & 0.892 & 61.2 & 1.11 \\
\hline 1024 & $1.024 \times 10^{6}$ & 660.0 & 1078.0 & 86.1 & 531.8 & 43.5 & 31.9 & 1.76 & 58.6 & 2.15 \\
\hline
\end{tabular}

Table 2: Comparison of iterations and computation time to solution in seconds on long domains with randomly generated right hand sides for the four methods averaged over ten trials of randomly generated right hand sides. In all cases, $\left(n, m_{z}\right)=(10,10)$, the GMRES tolerance was $10^{-10}$, and all were run on $32 \mathrm{MPI}$ ranks on a 4 processor 64 -core AMD Opteron $6366 \mathrm{HE}$.

\subsection{Increasing local problem size}


The previous two sections examined the effects of changing the domain size by either increasing the domain and element aspect ratio, or increasing domain aspect ratio while keeping element aspect ratio constant. In both cases, the number of grid points within each subdomain $\Omega_{j}$ was not changed. In this section, the effect of changing the local problem size on the convergence properties of the deflated-preconditioned Schur method is examined. For all of the following problems, the domain was a box $\Omega=[0,10] \times[0,10] \in \mathbb{R}^{2}$. The initial mesh was constructed with $n=5$ (polynomial order $p=4)$ and 4 elements in $x$ and $z\left(m_{x}=m_{z}=4\right.$ ). Denoting each element in the mesh as $V_{i j}$ with $i \in\left\{1, \ldots, m_{z}\right\}$ and $j \in\left\{1, \ldots, m_{x}\right\}$, recall that a subdomain in this decomposition is $\Omega_{j}=\bigcup_{i} V_{i j}$, implying $n^{2} m_{z}$ grid points per subdomain.

In the following three subsections, the number of grid points within each subdomain is increased by refining the mesh in three ways. First, the polynomial order $p=n-1$ is increased. Second, the number of elements in the vertical, $m_{z}$, is increased. Finally, the number of horizontal elements in each subdomain is increased by including multiple columns of elements in each subdomain. In all cases ten trials of each solver are computed and the average number of iterations required to achieve a relative residual of $10^{-10}$ is reported. The two-level block-Jacobi preconditioner is not tested since these grids are small and the coarse solver does not improve convergence.

\subsubsection{Polynomial order}

\begin{tabular}{|c|c|c|c|c|c|c|c|}
\hline & $p=4$ & $p=8$ & $p=12$ & $p=16$ & $p=20$ & $p=24$ & $p=28$ \\
\hline Schur & 20.0 & 31.8 & 42.9 & 54.4 & 65.9 & 76.0 & 84.5 \\
\hline BJ-Schur & 8.7 & 8.0 & 7.9 & 8.0 & 8.0 & 8.0 & 8.0 \\
\hline DBJ-Schur & 8.0 & 7.0 & 7.0 & 7.0 & 7.0 & 7.0 & 7.0 \\
\hline
\end{tabular}

Table 3: Number of iterations to achieve a tolerance of $10^{-10}$ averaged over ten trials as a function of $p$, the polynomial order, with three different preconditioning methods.

High-order element methods like the SMPM are in a sense $p$-version finite elements in that $p$, the polynomial order, can be increased while leaving $h$, the element size, unchanged. Thus, preconditioning strategies that are amenable to polynomial order refinement are desirable, and have been constructed before in the context of computational fluid dynamics [27]. To measure the performance of the deflation-preconditioning strategy as $p$-refinement takes place the initial grid with $m_{x}=m_{z}=4$ is made iteratively finer; the polynomial order is increased from $p=4$ to $p=28$. On each of seven grids spanning the space of $p \in[4,28]$ ten trials of deflation-preconditioned Schur are computed and the average number of iterations to achieve relative residual of $10^{-10}$ are reported in Table 3. Notice that although solving the Schur complement alone is not scalable with $p$, the use of the non-overlapping block-Jacobi preconditioner effectively removes the dependence of GMRES convergence on $p$. Use of deflation does not change this $p$-independence but also does not improve convergence significantly since there are only a few mesh elements in this grid. The observation, then, is that the block-Jacobi preconditioned Schur complement approach to solving the Poisson problem yields convergence independent of the polynomial order.

\subsubsection{Number of elements in $z$ per subdomain}

\begin{tabular}{|c|c|c|c|c|c|}
\hline & $m_{z}=4$ & $m_{z}=8$ & $m_{z}=16$ & $m_{z}=32$ & $m_{z}=64$ \\
\hline Schur & 20.0 & 18.8 & 18.1 & 20.5 & 23.0 \\
\hline BJ-Schur & 8.7 & 8.0 & 8.0 & 8.3 & 8.8 \\
\hline DBJ-Schur & 8.0 & 7.0 & 7.0 & 7.7 & 8.0 \\
\hline
\end{tabular}

Table 4: Number of iterations to achieve a tolerance of $10^{-10}$ averaged over ten trials as a function of $m_{z}$, the number of $z$ elements, with three different preconditioning methods.

Now, consider increasing the number of elements along the thin, $z$, direction. Since the domain decomposition is along $x$ alone, increasing the number of elements in the $z$ direction has the effect of increasing the number of grid points in a subdomain while leaving constant the number of interfaces and subdomains. In this study, the $4 \times 4$ element grid was $h$-refined by increasing $m_{z}$ from 4 to 64 while leaving $p=4, m_{x}=4$ and the domain $\Omega=[0,10] \times[0,10]$ unchanged. Ten trials of each of three preconditioning methods were solved on five grids spanning the space of $m_{z} \in[4,64]$, and the average number of iterations to achieve a $10^{-10}$ relative residual are reported in Table 4 While the unpreconditioned system shows some slight sensitivity to increasing $m_{z}$, neither the preconditioned nor the deflated preconditioned methods appear to have any such dependence. 
This is reasonable, since although the Schur problem grows linearly with $m_{z}$, its block structure does not change, and refinement in $m_{z}$ does not affect the element size or number along the decomposition direction.

\subsubsection{Number of elements in $x$ per subdomain}

\begin{tabular}{|c|c|c|c|c|c|c|c|}
\hline & $H / h=4$ & $H / h=8$ & $H / h=16$ & $H / h=32$ & $H / h=64$ & $H / h=128$ & $H / h=256$ \\
\hline Schur & 19.9 & 25.4 & 25.0 & 34.6 & 63.9 & 88.4 & 95.0 \\
\hline BJ-Schur & 8.6 & 11.5 & 11.0 & 12.0 & 17.0 & 23.0 & 28.0 \\
\hline DBJ-Schur & 8.0 & 10.8 & 10.1 & 11.0 & 23.0 & 29.0 & 35.0 \\
\hline
\end{tabular}

Table 5: Number of iterations to achieve a tolerance of $10^{-10}$ averaged over ten trials as a function of $w$, the number of $x$ elements per subdomain, with three different preconditioning methods. In this study the $H=L_{x} / 4$ is held constant, and $w$ is increased from 1 to 64 , with $H / h=w L_{x} / m_{x}$ where $L_{x}$ is the length of domain in $x$, and $m_{x}$ is the total number of elements in $x$.

To grow the local problem size in $m_{x}$, the subdomains in the domain decomposition are redefined to be multiple vertical strips of elements. This definition of subdomains is in contrast to the decomposition described in Section 2.3 and is being used here just to demonstrate the performance of the preconditioning methods for slightly more more general subdomains. Denoting as $w$ the number of vertical strips in each subdomain, and $V_{i j}$ an element in the $2 \mathrm{D}$ element grid, the first such subdomain $\Omega_{1}$ would be defined as

$$
\Omega_{1}=\bigcup_{j=1}^{w} \bigcup_{i=1}^{m_{z}} V_{i j} .
$$

Given the rectangular domain $\Omega=\left[0, L_{x}\right] \times\left[0, L_{z}\right]$, in the language of domain decomposition the subdomain width is now given by $H=w L_{x} / m_{x}$ and the element width is still $h=L_{x} / m_{x}$. Thus, by increasing $w$, the number of vertical strips of elements in each subdomain, the ratio $H / h$ is increased. In this study, the number of subdomains was held constant at four, and the initial mesh and domain was as in the previous two studies $\left(m_{x}=m_{z}=p=4\right.$ and $\left.L_{x}=L_{z}=10\right) . m_{x}$ was increased from 4 to 256 along with $w$ which was increased from 1 to 64 . This means that $H$ was fixed at $L_{x} / 4$, and $h$ was iteratively reduced from $h=L_{x} / 4$ to $h=L_{x} / 256$.

The simulation results are summarized in Table 5 , data which again represents the average number of GMRES iterations over ten trials of each of the three preconditioning methods. In summary, all three preconditioning methods show a gradual increase in the number of required iterations as $H / h$ becomes large. The block-Jacobi preconditioner reduces the number of iterations required to obtain a solution, and deflation worsens the convergence properties, likely because the coarse solution is not helpful when only four subdomains are present. While not logarithmic, the growth in the number of iterations appears to be very slow, approximately quadrupling as the local problem size grows by a factor of 64 .

It should also be mentioned that in the framework of a high-order discretization, in practice $w$ will be kept very small, usually equal to one. This is because the local element stiffness matrices $A$ have dense blocks whose dimensions scale with $p^{2}$. Therefore, if $w$ is allowed to grow, the work to solve the local problem on a processor quickly becomes prohibitive, and so it is preferable from the perspective of parallelization to keep $w$ small. Besides which, since it has been demonstrated in Section 4.3.1 that the convergence properties of this preconditioned Schur complement method are resilient to increasing the polynomial order, $p$-refinement is preferred to $h$-refinement, at least within the limitations imposed by the ill-conditioning of high-order numerical differentiation [14].

\subsection{Subdomain aspect ratio}

All the preconditioning methods described in this paper exhibit poor algorithmic scaling properties as the ratio of subdomain width $L_{x} / m_{x}$ to subdomain height $L_{z}$ is reduced. Since in the vertical-strip decomposition (c.f. Section 2.3) of the element grid each subdomain has aspect ratio $\eta_{\text {sub }}=\frac{L_{x}}{L_{z} m_{x}}$, subdomain aspect ratio is small if the domain aspect ratio $\left(L_{x} / L_{z}\right)$ is small and the number of elements in $x$ is large $\left(m_{x} \gg 1\right)$. To demonstrate the properties of the deflated block-Jacobi preconditioning method as $\eta_{\text {sub }}$ becomes small, the Schur problem was solved on three domains with aspect ratios of $10,10^{2}$, and $10^{3}$, and for grids with the number of $x$-elements growing from $m_{x}=4$ to $m_{x}=256$. $p=4$ and $m_{z}=4$ were fixed, and 10 trials of each preconditioner were solved with a required relative residual of $10^{-10}$. The number of GMRES iterations against the subdomain aspect ratios on the three domains and with all four preconditioners are shown in Fig. 7. 

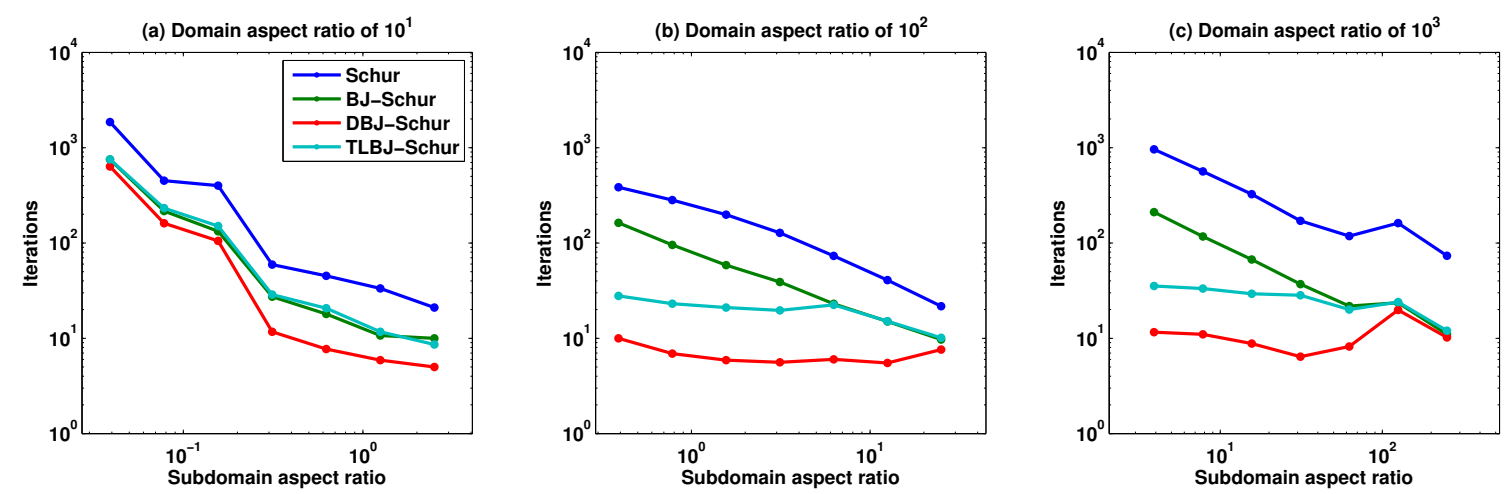

Figure 7: The average number of GMRES iterations to converge to a relative residual of $10^{-10}$ over ten trials of four types of preconditioners for the Schur complement problem over as a function $\eta_{\text {sub }}=L_{x} / m_{x}$, the subdomain aspect ratio, for three different domains. Left: A domain with $L_{x}=10 L_{z}$ and $p=m_{z}=4$. Middle: A domain with $L_{x}=100 L_{z}$ and $p=m_{z}=4$. Right: A domain with $L_{x}=1000 L_{z}$ and $p=m_{z}=4$.

Notice that as $\eta_{\text {sub }}$ becomes smaller that the number of iterations grows near uniformly on all three domains and for all preconditioners. While the deflated block-Jacobi preconditioner maintains the best performance, on the narrowest domain (Fig. 7(a)) its performance also degrades, barely outperforming the unpreconditioned Schur method. This study shows that there is a regularity constraint on the subdomain aspect ratio and, thus, the subdomain grid, namely that

$$
\mathrm{L}_{\mathrm{x}}>\mathrm{L}_{\mathrm{z}} \mathrm{m}_{\mathrm{x}}
$$

In the case of long domains in which $L_{x} \gg L_{z}$ decomposing into subdomains that are themselves vertical strips of elements is acceptable so long as this criteria is satisfied. If the subdomain aspect ratio grows too small, it may be necessary to either include more strips in each subdomain (as in Section 4.3.3), or to decompose along the horizontal as well as vertical inter-element interfaces in order to maintain an acceptable subdomain aspect ratio.

\section{Extension to three dimensions}

All of the preceding discussion has been strictly two-dimensional. A straightforward extension of the deflation method and domain decomposition to the three-dimensional problem is possible if it is assumed that the solution $u(x, y, z)$ is periodic in the $y$ direction, and that the domain is constant in the $y$ direction. The first assumption allows for a Fourier discretization in the transverse $y$ direction, and the second assumption allows for the re-use of the domain decomposition outlined in Section 2.3 in the three-dimensional case. In this section is a discussion of the construction and solution of the three-dimensional Poisson-Neumann Schur complement problem with a Fourier transverse discretization.

Denoting as $\Omega \subset \mathbb{R}^{2}$ a two-dimensional domain, denote as $\Omega^{\prime}=\Omega \times\left[0, L_{y}\right] \subset \mathbb{R}^{3}$ the domain on which the three-dimensional Poisson equation is to be solved. Assume that the boundary conditions in the third dimension $y \in\left[0, l_{y}\right]$ are periodic, implying a periodic solution $u(x, y=0, z)=u\left(x, y=L_{y}, z\right)$, and write the Poisson problem as

$$
\begin{aligned}
\nabla^{2} u & =f \text { on } \Omega^{\prime} \\
n \cdot \nabla u & =g \text { on } \partial \Omega \times\left[0, \mathrm{~L}_{y}\right], \\
u(x, 0, z) & =u\left(x, \mathrm{~L}_{y}, z\right) \text { on } \Omega \backslash \partial \Omega \times\left\{0, \mathrm{~L}_{y}\right\} .
\end{aligned}
$$

This type of extension from two to three dimensions facilitates a Fourier discretization in the $y$ dimension, and has been used previously [17, 38] in the context of solving the Navier-Stokes equations on problems and domains amenable to transverse periodicity of the solution. Admittedly requiring periodicity in the third dimension is a limitation and thus this is not a general three-dimensional formulation. However, as is the case in oceanic and atmospheric modeling of turbulence, only a transect of the large physical domain is discretized computationally, and the transverse direction is assumed to be the direction in which turbulence is statistically homogeneous and thus periodicity can be safely assumed. While restrictive, a periodic transverse direction still has significant applicability. 


\subsection{Construction of the three-dimensional Schur problems}

Starting with the problem in Eq. (30), force periodicity of the solution $u(x, y, z)$ in $y$ by taking the expansion of $u$ in the Fourier basis,

$$
u(x, y, z)=\sum_{j=0}^{m_{y} / 2-1} \hat{u}_{j}(x, z) e^{i k_{j} y}
$$

where $k_{j}=2 \pi j / h_{y}$ is the transverse wavenumber, $h_{y}=L_{y} / m_{y}$ spacing of the uniform grid in the transverse direction, $m_{y}$ the number of grid points in the transverse direction, and $\hat{u}_{j}(x, z) \in \mathbb{C}$ the Fourier coefficients. Substituting this Fourier expansion into Eq. (30), for each $k_{j}$ wavenumber a two-dimensional Helmholtz equation in $x$ and $z$ is obtained,

$$
\nabla^{2} \hat{u}_{j}(x, z)-k_{j}^{2} \hat{u}_{j}(x, z)=\hat{f}_{j}(x, z)
$$

where $\hat{u}_{j}, f_{j}$ are the $k_{j}$-th wavenumber components of the Fourier transforms of $u, f$ along the $y$ direction,

$$
\begin{aligned}
\hat{u}_{j} & =\hat{u}\left(x, k_{j}, z\right) \\
\hat{f}_{j} & =\hat{f}\left(x, k_{j}, z\right),
\end{aligned}
$$

and $\hat{u}\left(x, k_{y}, z\right)=\mathcal{F}_{y} u(x, y, z), \hat{f}\left(x, k_{y}, z\right)=\mathcal{F}_{y} f(x, y, z)$, where $\mathcal{F}_{y}$ is the discrete Fourier transform in $y$.

A complete description of the addition of a third Fourier dimension for the spectral multidomain penalty and spectral element methods can be found in Refs. 17] and [38 respectively, but the important fact is that all of the Schur complement methodology described thus far applies directly to each $k_{j}$ wavenumber in Eq. (32). This can be seen by writing the discrete version of Eq. (32) for the $j$-th wavenumber as

$$
\left(L-k_{j}^{2} I\right) \hat{u}_{j}=\hat{f}_{j}
$$

where now $u_{j}, f_{j} \in \mathbb{C}^{k}$ and $L$ is the SMPM Poisson-Neumann operator. Using the decomposition of $L=A+E B$ again write

$$
\left(A-k_{j}^{2} I\right) \hat{u}_{j}+E B \hat{u}_{j}=\hat{f}_{j}
$$

This is a Helmholtz equation with block-diagonal component $A-k_{j}^{2} I$ and off-diagonal component $E B$. Denoting the shifted block-diagonal matrix $A\left(k_{j}\right)=A-k_{j}^{2} I$, the Schur complement problem for wavenumber $k_{j}$ is given by

$$
S\left(k_{j}\right)=I+B A\left(k_{j}\right)^{-1} E,
$$

analogous to the unshifted case considered in Section 2.3 .

There are two important things to notice about Eq. (37). First note that while the $B$ matrix can depend on $\tau$, the penalty parameter, and in turn $\tau$ can depend on the shift $k_{j}^{2}$, acceptable values of $\tau$ span a broad range [34. It is possible to choose a $\tau$ that is suitable for all wavenumbers to make $B$ independent of wavenumber. Second, note that a division by $A-k_{j}^{2} I$ is required for each wavenumber $k_{j}$, and $A-k_{j}^{2} I$ certainly depends on wavenumber although only along its diagonal. To avoid operations of cubic complexity in computing $\left(A-k_{j}^{2}\right)^{-1}$, or calculating and storing separate factorizations of each $A\left(k_{j}\right)$, we appeal to the Schur factorization (not to be confused with a Schur complement). By computing and storing just the Schur factorization of $A(0)=U T U^{*}$, divisions by $A\left(k_{j}\right)$ for all $j$ can be solved in quadratic time. Since the unshifted $A(0)$ is block diagonal, its Schur factorization $U T U^{*}$ can be computed quickly in parallel. Noting that in a Schur factorization $U$ is unitary and $T$ upper triangular, the Schur factorization of $A\left(k_{j}\right)$ for any $k_{j}$ is given by

$$
\begin{aligned}
A\left(k_{j}\right) & =A-k_{j}^{2} I \\
& =U T U^{*}-k_{j}^{2} I \\
& =U\left(T-k_{j}^{2} I\right) U^{*} .
\end{aligned}
$$


Because $A(0)$ is block-diagonal, $U$ is block unitary and $T-k_{j}^{2} I$ is block upper triangular, so any division $x=A\left(k_{j}\right) \backslash b$ is solvable in parallel and in quadratic time by

$$
x=U\left(T-k_{j}^{2}\right)^{-1} U^{*} b
$$

where $\left(T-k_{j}^{2} I\right)^{-1}$ is a block back-substitution and multiplications by $U$ and $U^{*}$ are block matrix-vector multiplications. Both of these operations are parallel over the blocks of $A$ and are of quadratic complexity. Finally, it is worth noting that although $A\left(k_{j}\right) \in \mathbb{R}^{r \times r}$ is real, its Schur factors $U, T \in \mathbb{C}^{r \times r}$ are in general complex, which roughly doubles the storage cost. From the perspective of domain decomposition, the Schur factorization is a powerful tool. It allows for solving linear systems in $A\left(k_{j}\right)$ in quadratic time while only requiring the Schur factorization and storage of $A(0)$. The Schur factorization of $A(0)$ is furthermore computable in parallel since $A(0)$, and consequently $U$ and $T$ are block-diagaonal.

After computing $A(0)=U T U^{*}$, each transverse Schur problem is assembled as

$$
\begin{aligned}
S\left(k_{j}\right) \hat{x}_{j} & =\hat{b}_{j} \\
\left(I+B A\left(k_{j}\right)^{-1} E\right) \hat{x}_{j} \hat{x}_{j} & =\hat{b}_{j} \\
\left(I+B U\left(T-k_{j}^{2} I\right) \backslash U^{*} E\right) \hat{x}_{j} & =\hat{b}_{j},
\end{aligned}
$$

where $\hat{x}_{j}=B \hat{u}_{j}$ and $\hat{b}_{j}=B A\left(k_{j}\right)^{-1} \hat{f}_{j}$. Then, each transverse wavenumber's solution $\hat{u}_{j}$ is obtained from the solution of the Schur problem $\hat{x}_{j}$ as

$$
\begin{aligned}
& \hat{u}_{j}=A\left(k_{j}\right)^{-1}\left(\hat{f}_{j}-E \hat{x}_{j}\right) \\
& \hat{u}_{j}=U\left(T-k_{j}^{2} I\right)^{-1} U^{*}\left(\hat{f}_{j}-E \hat{x}_{j}\right),
\end{aligned}
$$

and the full three-dimensional solution is assembled via the inverse Fourier transform,

$$
u(x, y, z)=\sum_{j} \hat{u}_{j}(x, z) e^{i k_{y} y}
$$

Each Schur problem $S\left(k_{j}\right) \hat{x}_{j}=\hat{b}_{j}$ can be solved in parallel with the same deflation preconditioning technique demonstrated previously. The assembly of each Schur matrix $S\left(k_{j}\right)$ is non-trivial, but is amortized over the many Poisson solves required in a time-evolving Navier-Stokes solver.

The deflated and preconditioned three-dimensional Schur problem is formulated as a sequence of independent two-dimensional Schur problems, each a Helmholtz equation representing the $k_{j}$ wavenumber component of the solution $u(x, y, z)$. Identical to the formulation of the zero wavenumber two-dimensional problem in Eq. 25), the deflated and preconditioned problems are for each $k_{j}$

$$
P\left(k_{j}\right) S\left(k_{j}\right) M\left(k_{j}\right) \hat{x}_{j}=P\left(k_{j}\right) B A\left(k_{j}\right)^{-1} \hat{b}_{j}
$$

where $M\left(k_{j}\right)$ is the block-Jacobi preconditioner of $S\left(k_{j}\right), P\left(k_{j}\right)=I+S\left(k_{j}\right) Z C\left(k_{j}\right)^{-1} Z^{T}$, and $C\left(k_{j}\right)=$ $Z^{T} S\left(k_{j}\right) Z$. The solution for wavenumber $k_{j}$ is obtained analogously to that in Eq. 25 as

$$
\begin{aligned}
\hat{x_{j}} & =Z C\left(k_{j}\right) Z^{T} \hat{b}_{j} \\
& +Q\left(k_{j}\right) M\left(k_{j}\right)^{-1} \operatorname{GMRES}\left(P\left(k_{j}\right) S\left(k_{j}\right) M\left(k_{j}\right)^{-1}, P\left(k_{j}\right) \hat{b}_{j}\right) .
\end{aligned}
$$

where $Q\left(k_{j}\right)=I+Z C\left(k_{j}\right)^{-1} Z^{T} S\left(k_{j}\right)$. The zero wavenumber problem $k_{j}=0$ requires additional regularization as has been extensively documented in Section 2.4 .

\subsection{Implementation}

While each wavenumber's Schur complement problem is independent of the others, in practice, all of the wave numbers are solved simultaneously in one GMRES calculation. This is done to minimize the communication overhead inherent in computation of dot products that plagues all Krylov methods [30; by solving all of the Schur problems together, the communication overhead penalty is paid only once per Krylov iteration instead of $m_{y}$-many times.

The algorithmic summary of this method is given in Algorithm 4, which presumes that an initial setup phase has been conducted to compute the Schur factorization of $A(0)$, the assembly of $S\left(k_{j}\right)$ for $j=1, \ldots, m_{y}$, the block-Jacobi preconditioner $M\left(k_{j}\right)$, and the coarse grid matrix $C\left(k_{j}\right)$. Since the 


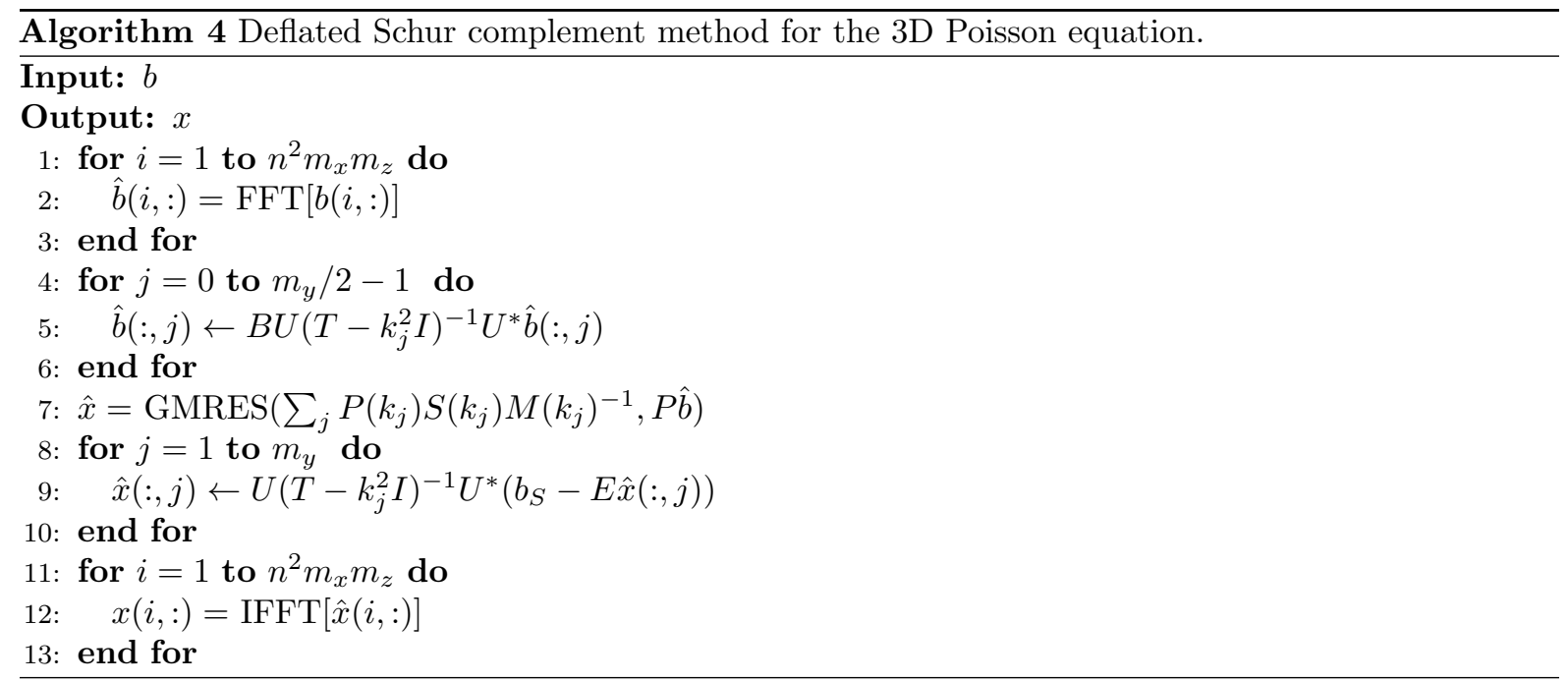

two-dimensional problem corresponding to the $k_{j}=0$ wavenumber is rank-deficient, a regularization identical to that in Algorithm 2 is done for $k_{y}=0$ which is not shown in Algorithm 4.

The two for-loops wrapping the fast Fourier transforms (FFTs) are embarrassingly parallel and in practice multi-threaded ${ }^{3}$. The divisions by $A\left(k_{j}\right)$ (lines 5 and 9 ) are block-solves each of quadratic complexity, and so are negligible relative to the expensive GMRES iteration for the solution of the three-dimensional Schur complement system. When appropriately parallelized on $N_{p}$ CPU cores, the number of floating point operations per rank for the above algorithm is

$$
\begin{aligned}
F & =\overbrace{2 n^{2} m_{z} \frac{m_{x}}{N_{p}} \mathcal{O}\left(m_{y} \log m_{y}\right)}^{n^{2} m_{z} m_{x} / N_{p} \text { FFTs and IFFTs }} \\
& +\underbrace{6 m_{y} \frac{m_{x}}{N_{p}} \mathcal{O}\left(\left(n^{2} m_{z}\right)^{2}\right)}_{6 m_{y} m_{x} / N_{p} \text { Schur Solves }}+\underbrace{\frac{m_{x}}{N_{p}} \mathcal{O}\left(K^{3} 16 m_{y} n^{2} m_{z}^{2}\right)}_{1 \text { GMRES solve of } K \text { iterations }}
\end{aligned}
$$

where $K$ is the number of GMRES iterations required for a solve. In practical applications $m_{x} / N_{p}$ is kept bounded, and so if $K$ depends on either $m_{z}$ or $n$, then the third term will quickly become dominant due to its cubic dependence on $K$. Thus, even in the three-dimensional case, minimizing GMRES iterations $K$ is still the most important aspect of achieving good performance.

\subsection{Performance}

To study the performance of the three-dimensional solver, the two-dimensional simulations represented in Table 2 were extended by extruding the two-dimensional domain into $\Omega \times\left[0, l_{y}\right]$, with $m_{y}$ grid points in the transverse direction. The right hand sides used are again randomly drawn from a uniform distribution to ensure that their Fourier transforms will have significant components in all wavenumbers $k_{j}$. As before, the number of elements in $x$ was increased from $m_{x}=64$ to $m_{x}=1024$ while keeping their aspect ratio constant, while the other parameters $\left(n, m_{y}, m_{z}\right)=(10,32,10)$ were held constant, resulting in grids ranging from 2 to 32 million grid points in size. From Table 2 only the deflation and two-level block-Jacobi preconditioners were used, as the other two methods required too much computation time and Krylov iterations to be of practical use. All results were computed on 32 MPI ranks, and all results were averaged over 10 trials.

The results comparing the deflated block-Jacobi preconditioning with two-level block-Jacobi preconditioning are displayed in Table 6. First notice that the number of GMRES iterations does not increase substantially from the two-dimensional problems (Table 2). This is primarily because the worst-conditioned 
of all the wavenumbers is the $k_{0}=0$ wavenumber which is what is solved in the two-dimensional problems; adding more wavenumbers only adds better conditioned problems that converge earlier than the zero wavenumber. Second, note that the deflation method again outperforms two-level block-Jacobi preconditioning, requiring about $40 \%$ fewer GMRES iterations and $15 \%$ less time. The difference in iterations does not translate directly into time because again the application of $P$ in the deflation method adds an extra multiplication of $S$ to each GMRES iteration. While, from one perspective, deflation is only modestly faster than two-level preconditioning, when solving the Poisson equation hundreds if not thousands of times within a time-evolving fluid simulation, even a $15 \%$ improvement in time translates to significant savings in total time. For extremely large problems where storage of Krylov basis vectors is a significant cost the reduction in GMRES iterations in deflated block-Jacobi preconditioning relative to two-level block-Jacobi preconditioning can be important.

\begin{tabular}{|rrr|lr|rr|}
\cline { 4 - 7 } \multicolumn{1}{l|}{} & & \multicolumn{2}{l|}{ Deflated block-Jacobi } & \multicolumn{2}{c|}{ TL block-Jacobi } \\
$m_{x}$ & Grid Points & Setup Time & Iter. & Time & Iter. & Time \\
\hline 64 & $2.05 \times 10^{7}$ & 75.0 & 30.5 & 2.30 & 48.1 & 2.71 \\
128 & $4.10 \times 10^{7}$ & 152.0 & 57.0 & 7.21 & 84.1 & 8.29 \\
256 & $8.19 \times 10^{7}$ & 313.0 & 39.2 & 10.8 & 60.0 & 12.3 \\
512 & $1.64 \times 10^{8}$ & 658.0 & 33.7 & 19.8 & 52.7 & 22.7 \\
1024 & $3.28 \times 10^{8}$ & 1500.0 & 31.8 & 52.1 & 50.4 & 65.0 \\
\hline
\end{tabular}

Table 6: Comparison between the 3D deflation method and the 3D two-level block-Jacobi method of iterations and computation time to solution in seconds. The number of GLL points per direction is $n=10$, the number of vertical elements $m_{z}=10$, and number of transverse wave numbers $m_{y}=32$. These simulations were all benchmarked in an MPI-parallel Fortran code and executed on $32 \mathrm{MPI}$ ranks on a 4 processor 64-core AMD Opteron 6366 HE.

Lastly, note that the third column in Table 6 shows the time taken to perform the assembly, factorization, and inverse iteration done in the setup phase. The time for setup is usually about 20 times the time taken for a single solve, which is easily amortized over the many tens of thousands of solves required in a simulation [17, 21].

\section{Discussion}

\subsection{Related methods and discretizations}

The SMPM was used here as the platform to demonstrate the effectiveness of the deflated block-Jacobi preconditioned Schur complement approach, but this approach can be extended to other discretizations as well. Domain decomposition has of course been applied to many different kinds of discretizations [58] including continuous [49] and discontinuous Galerkin, collocation [5], and spectral element methods [43. 15. While most domain decomposition methods separate the grid into internal and interface unknowns, the particular decomposition shown in Section 2.3 decomposes the discrete operator into intraand inter-subdomain components and solves for the inter-subdomain fluxes $B u$ first before solving the local block problems. As such, it represents an algebraic approach to decomposition, not unlike previous uses of the Sherman-Morrison-Woodbury identity to solve linear systems that are modified with a low-rank update.

Crucially, the discontinuous element nature of the SMPM makes the interior problems represented by $A$ all invertible and decoupled. Similar to the SMPM, discontinuous finite and spectral element methods have recently garnered more attention from the domain decomposition literature [3, 9, 11], as their non-conforming nature leads to stencil locality and the ability to build non-overlapping Schwarz preconditioners that still scale algorithmically as $H / h$ [25, 2]. By using a Schur complement approach, it is

shown here in Section 4.3.1 that for discontinuous discretizations a preconditioning approach that involves deflation also yields convergence essentially independent of polynomial order. For high-order discontinuous element methods, this seems to suggest that the Schur complement approach derived here is preferable. While superficially resembling the FETI-DP [22] or the BDDC [41] methods in that primal constraints are analogous to the inter-element fluxes $B u$ (c.f. Section 2.3), since no presumption of inter-element continuity is made in the SMPM Schur complement method there is no need for distinguishing between interface and interior unknowns or taking care to ensure conformity between domains. Since no nodal point in the SMPM discretization lies in more than one element, no nodal point lies in more than one subdomain, so cross-points are also not problematic. Finally, some work has been done recently to extend FETI-DP [24] and BDDC preconditioners [11] to discontinuous discretizations showing convergence independent of the number of subdomains and weakly dependent on $p$ and $H / h$. 
Because of its similarities, the SMPM Schur complement approach taken here is extensible to discontinuous Galerkin discretizations, and is especially promising for high-order elements. The discontinuous stencil is necessary to ensure a block-diagonal interior problem matrix $A$ in the derivation in Section 2.3. and high-order polynomials lead to a Schur interface grid whose density is small in the full grid. The solution to the Schur complement problem would represent the interfacial fluxes in the DG residual, but otherwise algebraically the derivation in Section 2.3 would proceed identically. Since discontinuous Galerkin methods are widely used, this seems like a natural application for deflation-augmenting preconditioning of the Schur complement problem.

Finally, while this paper focused solely on the Poisson-Neumann problem due to its relevance in the problem of interest, the approach taken extends to any elliptic problem.

\subsection{Overlapping block-Jacobi preconditioners}

Commonly in additive-Schwarz-type preconditioning methods subdomains are chosen to be overlapping. For preconditioning the full operator, it has been shown that to achieve grid-independent convergence subdomain overlap is necessary [27, 47, with the amount of overlap scaling with the size of the subdomains. Since all of the preconditioning methods demonstrated here are applied to the Schur complement system and not the full system, a reasonable question to ask might be whether overlapping the blocks of the block-Jacobi preconditioner $M$ improves convergence in the Schur complement system.
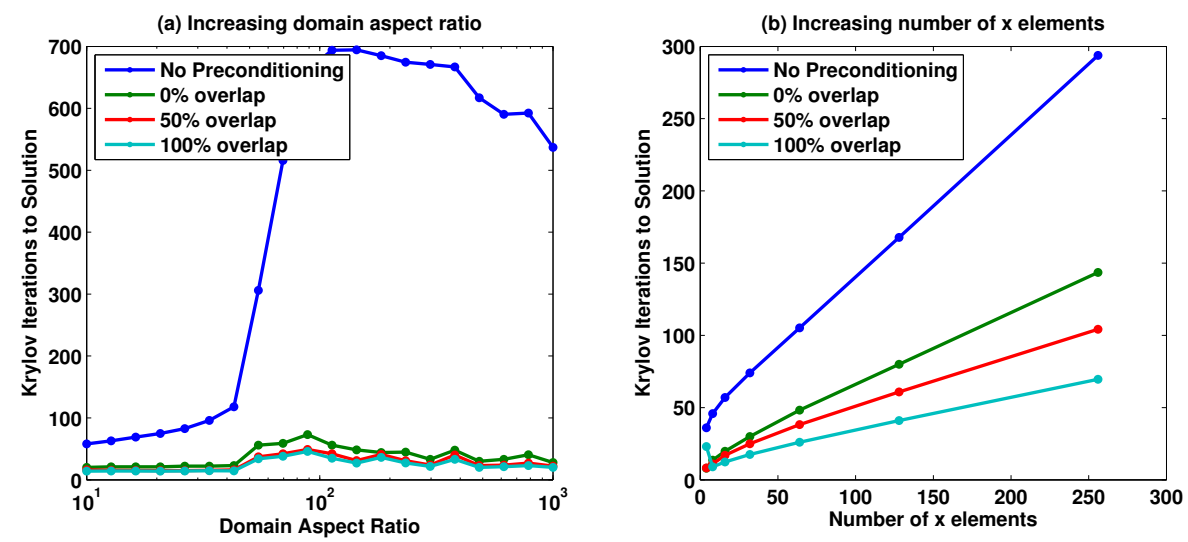

Figure 8: A study of the effect of increasing overlap of the blocks of the Schur complement block-Jacobi preconditioner. Left: The number of GMRES iterations to achieve a relative residual of $10^{-10}$ as the domain and element aspect ratio is increased. Right: The number of GMRES iterations to achieve a relative residual of $10^{-10}$ as the number of elements in the $x$ direction is increased.

To study this, the block-Jacobi preconditioner for the Schur complement was modified to include overlapping blocks in a purely algebraic fashion (the overlap does not correspond to subdomain overlap since the blockJacobi preconditioner is applied to the Schur grid). Since with no overlap the blocks of the block-Jacobi preconditioner correspond to the interfaces along the boundaries of a subdomain (c.f. Figs. 2 and 3), 25\%, $50 \%$, and $100 \%$ overlap correspond to increasing the number of interfaces included in one block. For each of these three values of overlap, problems with increasing domain aspect ratio and number of elements in the long direction were solved. The average number of iterations to solution for increasing aspect ratio are reported in Fig. 8(a), and the average number of iterations to solution for increasing $m_{x}$ are reported in Fig. 8(b). Evident from both of these plots is that although increasing the fraction of overlap does in fact reduce the number of iterations required for solution, the asymptotic behavior is the same as including no overlap. This is to say that the inclusion of overlap does not mitigate the growth in GMRES iterations as $m_{x}$ is increased. This is very much unlike preconditioning the full problem, in which good asymptotic behavior of the preconditioner is contingent on subdomain and consequently block overlap [27], especially in the case of increasing polynomial order [47]. That the non-overlapping preconditioner in this Schur complement problem is only slightly less effective than the overlapping versions implies that as a matter of practicality it is not worth incurring the significant additional inter-processor communication overhead of using overlapping blocks, and so our preconditioning strategy avoids it. 


\section{Summary and Concluding Remarks}

In this paper, a method of solving the Poisson-Neumann problem on large, highly-elongated domains such as those that are common in the computational simulation of non-hydrostatic environment fluid phenomena is presented. In particular, a method of preconditioning the Schur complement problem of the twodimensional Poisson-Neumann system discretized by a high-order discontinuous element-based collocation method has been developed. The large, highly-elongated grids introduce two difficulties to solving the Poisson-Neumann system. First, the highly-stretched grids lead to ill-conditioned spectral differentiation matrices which are addressed by way of a block-Jacobi preconditioner on the Schur complement problem. Second, for grids with many elements, Krylov subspace methods require many iterations to communicate information across the grid which is addressed by augmenting the block-Jacobi preconditioner with a set of deflation vectors that eliminates the residual in a coarse subspace of the Schur complement matrix. Since the Schur complement problem is better conditioned and a factor of $n$ smaller than the full Poisson problem, it requires fewer Krylov iterations and less storage for the Krylov basis vectors.

It is shown that by using deflation-augmented block-Jacobi preconditioning on the Schur complement, the number of Krylov iterations can be kept bounded as the aspect ratio (Section 4.1) is increased and as the number of subdomains is increased (Section 4.2). Furthermore, the method shows convergence independent of the polynomial order (Section 4.3.1) and number of $m_{z}$ elements (Section 4.3.2), and the number of iterations grow gradually with increasing number of elements per subdomain (Section 4.3.3). Comparisons with two-level block-Jacobi preconditioning for the same problem showed that deflation required approximately half as many Krylov iterations and required about $25 \%$ less time. It is shown in Section 5 that when a third dimension was added by way of a Fourier discretization, the superiority of deflation in two-dimensions translates to the three-dimensional problem as well; in three dimensions, the deflation preconditioned Schur complement problem requires $40 \%$ fewer Krylov iterations and 15\% less time than two-level block-Jacobi preconditioning.

The three main contributions of this work may be summarized as follows. First, the use of deflationaugmented block-Jacobi preconditioning on the Poisson-Neumann Schur system is shown to be a viable candidate technique for obtaining the solution to the Schur complement problem. The convergence of the GMRES calculation on the deflated and preconditioned Schur matrix is independent of the domain aspect ratio, the number of $x$ elements, and the polynomial order, all important criteria for a solution algorithm and the subject of much study [26, 27, 25]. Development of algorithms that satisfy these criteria has largely been focused on two-level block-Jacobi methods applied to the Poisson problem itself. While there admittedly exists a significant body of literature on using two-level block-Jacobi preconditioning for the Schur complement problem of elliptic equations [49, 5, 43, and also a significant body of literature on using deflation methods to accelerate convergence on elliptic problems themselves [61, 45, 55], applying deflation acceleration to the Schur complement problem seems largely unexplored. It is also worth noting that the SMPM discretized Poisson-Neumann operator is unsymmetric, and some care was taken to show that the projections out of the null space of this rank-deficient operator did not affect the quality of the solution. While certainly most discretizations of the self-adjoint Poisson equation are symmetric positive definite themselves (as in the spectral element or discontinuous Galerkin methods), this also shows that, without much extra effort, unsymmetric discretizations can also be accounted for.

Secondly, it is demonstrated in Sections 4 and 5 that deflation acceleration alongside block-Jacobi preconditioning outperforms two-level block-Jacobi at least for the SMPM discretization of the PoissonNeumann problem. For both the two-dimensional (Section 4) and the three-dimensional (Section 5) case, deflated block-Jacobi preconditioning required about half as many GMRES iterations and 15\%-25\% less computation time than two-level block-Jacobi. While this is admittedly a limited comparison and a modest performance gain, it is in agreement with previous work comparing deflation with two-level block-Jacobi methods for preconditioning the full Poisson problem [45, 56, 57. In light of the fact that for many of these environmental-scale problems the duration of the computation is on the order of several months of wall-clock time [19], even a $15 \%$ reduction in computation time as shown in Section 5 can be significant. Considering also the $50 \%$ reduction in the memory footprint due to the $50 \%$ reduction in Krylov iterations (and thus basis vectors), it is possible that the performance difference between the deflation and the two-level block Jacobi methods may be significant.

Finally, use of the Schur factorization of the local block problems $A\left(k_{j}\right)$ is used to quickly factor and divide all the local element matrices in transverse direction when extending to three-dimensions (Section 5). Coupled with the domain decomposition outlined in Section 4 the use of the Schur factorizations allows for parallel division of the block-diagonal matrix $A\left(k_{j}\right)$ and the re-use of the Schur factorization 
of $A(0)=U T U^{*}$. Without the Schur factorization, either the factorization of each $A\left(k_{j}\right)$ would need to be stored or a different approach, such as Gaussian elimination, would be required to perform the divisions of $\left\{A\left(k_{j}\right)\right\}_{j=1}^{m_{y}}$. For grids with many transverse wavenumbers $\left(m_{y} \gg 1\right)$ the savings in storage and factorization time can be immense, and in practice $m_{y}$ is often much larger than the $m_{y}=32$ value used in Section 5. reaching from $m_{y}=128$ [17] to as high as $m_{y}=768$ [18] in environmental-scale applications. When $m_{y}$ is this large, storing a single $A(0)$ and computing a single Schur factorization instead of $m_{y}$-many is an important advantage of a Fourier-discretized transverse direction leveraging the Schur factorization.

\subsection{Future work}

Since the primary motivation for solving the Poisson equation in this work is to obtain a numerical solution to the incompressible Navier-Stokes equations, immediate interest is in implementing the deflated block-Jacobi Schur method in an incompressible Navier-Stokes solver. By way of the operator splitting as in Ref. [39], the Poisson problem is separated from the viscous and nonlinear advective terms which means the methodology developed in this work can be applied in a relatively straightforward manner.

Some extensions to the deflation block-Jacobi method described here might be to a fully threedimensional discretization or to an unstructured grid. As discussed in Section 4.4 two-dimensional or three-dimensional decompositions may be necessary in order to keep the subdomain aspect ratio amenable for these iterative algorithms. First, in a fully three-dimensional grid, the Schur complement would likely be too large too store, but otherwise the construction of its deflation vectors and its preconditioner would be an extension of the current aproach. For example if the Schur matrix was constructed by decomposing along all element interfaces, each block of the local matrix $A$ would be of dimension $n^{3}$, and the Schur complement matrix $S$ would be of dimension $6 n^{2}\left(m_{x}-1\right)\left(m_{y}-1\right)\left(m_{z}-1\right)$. Thus the Schur matrix would still be a factor of $n$ smaller than the full Poisson matrix, and would be better conditioned by virtue of being a Schur complement. Critically, because of the discontinuous nature of the SMPM, extensions to 2D and 3D decompositions would not involve cross-points, since no grid point would lie in more than one subdomain. If the grid were also unstructured the choice of subdomains becomes more complicated, but can be reduced to a graph partitioning problem that has received much attention as a computational problem [40. Thus, while requiring a significantly greater programming investment, the lack of cross points due to the non-conforming discontinuous discretization means that the extension of the Schur complement and deflation methods outlined is theoretically tractable, and a possible avenue for future development.

One open question worth investigating is related to the choice of deflation vectors $Z$ in Section 3.4 The deflation vectors were chosen to be discrete indicator vectors along the domain decomposition boundaries $\Gamma_{i}$ after a choice made in Section 4.1.1 of Ref. [57, a simulation of porous media flow that required the solution of Poisson-Dirichlet system of equations. This choice is sensible, as it approximates eigenvectors that represent modes of the Schur matrix in which all nodes along an interface $\Gamma_{i}$ have the same value; this represents low-order modes of oscillation in a sense, and is in the spirit of what deflation is attempting to do. However, we do not claim (or even suggest) that this choice of $Z$ is optimal, and it is likely that other deflation vectors and thus coarsening operators will provide better performance.

Lastly, although it is true that by replacing the boundary fluxes $B u$ with the inter-element fluxes in the discontinuous Galerkin method (DGM) the Schur complement assembly and deflation techniques can extend to high-order DGM discretizations, a demonstration of that claim with the aim of studying the relative performance of the SMPM against the DGM would be highly insightful.

\section{Acknowledgements}

The authors would like acknowledge Professors Charles Van Loan, Jorge Escobar-Vargas, and David Bindel for helpful comments on the work in this manuscript. The authors would also like to thank the United States Department of Defense High-Performance Computing Modernization Office for the NDSEG fellowship and the National Science Foundation for CAREER award Grant No. OCE-0845558 for support. Finally the authors would like to thank the reviewers for their insightful and very helpful comments on this manuscript. High performance computing resources were provided through the U.S. Department of Defense High Performance Computing Modernization Program by the Army Engineer Research and Development Center and the Army Research Laboratory under Frontier Project FP-CFDFY14-007, and by the Open Research Systems. 


\section{Appendix: proof of claims}

Claim 1. Denote as $u_{L} \in \mathbb{R}^{r}$ the left null vector of $L \in \mathbb{R}^{r \times r}$. Denote as $u_{S} \in \mathbb{R}^{k}$ the left null vector of $S \in \mathbb{R}^{k \times k}$. Then the following relations hold.
1. $u_{S}=E^{T} u_{L}$
2. $u_{L}=A^{-T} B^{T} u_{S}$

Proof. First, note that $u_{L}^{T}(A+E B)=0_{r} \in \mathbb{R}^{r}$ which implies that $u_{L}^{T}+u_{L}^{T} E B A^{-1}=0_{r}$. Right multiply by $E$ to obtain $u_{L}^{T} E+u_{L}^{T} E B A^{-1} E=0_{r}$ which yields the first relation that $u_{L}^{T} E\left(I+B A^{-1} E\right)=0_{r} \Rightarrow$ $u_{L}^{T} E S=0_{k} \Rightarrow u_{S}=E^{T} u_{L} \in \mathbb{R}^{k}$.

For the second part, note that $B A^{-1} L=B A^{-1}(A+E B)=B+B A^{-1} E B=S B$. Thus if $u_{S}^{T} S=0_{k}$, $u_{S}^{T} B A^{-1} L=0_{k}$, and so $A^{-T} B^{T} u_{S} \in \mathbb{R}^{r}$ is the left null vector of $L$.

Claim 2. Given $L u=\tilde{f} \in \mathbb{R}^{r}$, if $u_{L}^{T} \tilde{f}=0$, then the error in the solution $u=A^{-1}\left(\tilde{f}-E x_{S}\right)$ recovered from the solution of the regularized Schur complement system $S x_{S}=b_{S}-u_{S} u_{S}^{T} b_{S}$ is bounded by the error of Schur complement solution:

$$
\|L u-\tilde{f}\|_{2} \leq\left\|S_{x_{S}}-\left(I-u_{S} u_{S}^{\top}\right) b_{S}\right\|_{2}
$$

Proof. Start with the Poisson residual $L u-\tilde{f}$, substitute the solution $u=A^{-1}\left(\tilde{f}-E x_{s}\right)$, and recall that $L=A+E B$ to obtain

$$
\begin{aligned}
L u-\tilde{f} & =L A^{-1}\left(b-E x_{s}\right)-\tilde{f} \\
& =(A+E B) A^{-1}\left(\tilde{f}-E x_{S}\right)-\tilde{f} \\
& =\left(I+E B A^{-1}\right)\left(\tilde{f}-E x_{S}\right)-\tilde{f} \\
& =E B A^{-1} \tilde{f}-E S x_{S} \\
& =E\left(B A^{-1} \tilde{f}-S x_{S}\right) .
\end{aligned}
$$

If $S$ were full-rank, then $x_{s}=S^{-1} B A^{-1} \tilde{f}$ as calculated in domain decomposition and the right hand side would be zero. However due to the rank-deficiency of $S$ the residual that is made small in the Schur complement solve is $\left\|S x_{s}-\left(I-u_{S} u_{S}^{T}\right) B A^{-1} \tilde{f}\right\|_{2}$. So, adding and subtracting the projection to the above, we obtain a bound on the residual:

$$
\begin{aligned}
\|L u-\tilde{f}\|_{2} & =\left\|E\left(B A^{-1} \tilde{f}-u_{S} u_{S}^{T} B A^{-1} \tilde{f}+u_{S} u_{S}^{T} B A^{-1} \tilde{f}-S x_{s}\right)\right\|_{2} \\
& \leq\left\|E\left(B A^{-1} \tilde{f}-u_{S} u_{S}^{T} B A^{-1} \tilde{f}-S x_{s}\right)\right\|_{2}+\left\|E u_{S} u_{S}^{T} B A^{-1} \tilde{f}\right\|_{2} \\
& \left.\leq \|\left(I-u_{S} u_{S}^{T}\right) B A^{-1} \tilde{f}-S x_{s}\right)\left\|_{2}+\right\| E u_{S} u_{S}^{T} B A^{-1} \tilde{f} \|_{2} .
\end{aligned}
$$

The first term in the above is exactly the residual we minimize in the Schur complement solve; the second term is an error term related to projecting out of the column space of $S$. Examining the second term, recall from Claim 1 that $u_{S}=E^{T} u_{L}$, and substitute this into the expression for the second term:

$$
\begin{aligned}
\left\|E u_{S} u_{S}^{T} B A^{-1} \tilde{f}\right\|_{2} & =\left\|E\left(E^{T} u_{L}\right)\left(E^{T}\left(u_{L}\right)\right)^{T} B A^{-1} \tilde{f}\right\|_{2} \\
& =\left\|E\left(E^{T} u_{L}\right) u_{L}^{T} E B A^{-1} \tilde{f}\right\|_{2} .
\end{aligned}
$$

Recalling that $E B=L-A$ we get

$$
\begin{aligned}
& =\left\|E E^{T} u_{L} u_{L}^{T}(L-A) A^{-1} \tilde{f}\right\|_{2} \\
& =\left\|E E^{T} u_{L}\left(u_{L}^{T} L A^{-1} \tilde{f}-u_{L}^{T} \tilde{f}\right)\right\|_{2} .
\end{aligned}
$$


Now by definition $u_{L}^{T} L=0_{r}$ so the first term in the above is zero, and by assumption $u_{L}^{T} \tilde{f}=0_{r}$ so the second term is zero. Thus $\left\|E u_{S} u_{S}^{T} B A^{-1} \tilde{f}\right\|_{2}=0$, and we have our bound,

$$
\|L u-\tilde{f}\|_{2} \leq\left\|S x_{S}-\left(I-u_{S} u_{S}^{T}\right) b_{S}\right\|_{2},
$$

where $b_{S}=B A^{-1} \tilde{f}$.

\section{References}

[1] Abdilghanie, A. M., Diamessis, P. J., 2013. The internal gravity wave field emitted by a stably stratified turbulent wake. Journal of Fluid Mechanics 720, 104-139.

[2] Antonietti, P. F., Ayuso, B., 2007. Schwarz domain decomposition preconditioners for discontinuous Galerkin approximations of elliptic problems: non-overlapping case. ESAIM: Mathematical modeling and numerical analysis $41(1)$.

[3] Antonietti, P. F., Houston, P., 2010. A Class of Domain Decomposition Preconditioners for hp-Discontinuous Galerkin Finite Element Methods. Journal of Scientific Computing 46, 124-149. URL http://link.springer.com/10.1007/s10915-010-9390-1

[4] Beuchler, S., 2002. Multigrid solver for the inner problem in decomposition methods for p-FEM. SIAM J. Numer. Anal. $40(3), 928-944$.

[5] Bialecki, B., Karageorghis, A., 2007. A nonoverlapping domain decomposition method for Legendre spectral collocation problems. Journal of Scientific Computing 32 (2), 373-409.

[6] Bjorck, A., 1967. Solving linear least squares problems by Gram-Schmidt Orthogonalization. Bit 7, 1-21.

[7] Brenner, S. C., 1996. Two-level additive Schwarz preconditioners for nonconforming finite element methods. Mathematics of Computation 65 (215), 897-922.

[8] Brenner, S. C., 1999. The condition number of the Schur complement in domain decomposition. Numerische Mathematik 83, 187-203.

[9] Brix, K., Campos Pinto, M., Canuto, C., Dahmen, W., 2015. Multilevel preconditioning of discontinuous Galerkin spectral element methods. Part I: geometrically conforming meshes. IMA Journal of Numerical Analysis 35, 1487-1532. URL http://imanum.oxfordjournals.org/lookup/doi/10.1093/imanum/dru053

[10] Brown, P. N., Walker, H. F., 1997. GMRES On (Nearly) Singular Systems. SIAM Journal on Matrix Analysis and Applications 18 (1), 37-51.

[11] Canuto, C., Pavarino, L. F., Pieri, a. B., 2013. BDDC preconditioners for continuous and discontinuous Galerkin methods using spectral/hp elements with variable local polynomial degree. IMA Journal of Numerical Analysis 34, 879-903. URL http://imanum.oxfordjournals.org/cgi/doi/10.1093/imanum/drt037

[12] Carvalho, L. M., Giraud, L., Le Tallec, P., 2001. Algebraic Two-Level Preconditioners for the Schur Complement Method. SIAM Journal on Scientific Computing 22, 1987-2005.

[13] Cockburn, B., Karniadakis, G. E., Shu, C.-W. (Eds.), 2000. Discontinuous Galerkin Methods, 1st Edition. Springer Berlin Heidelberg.

[14] Costa, B., Don, W. S., 2000. On the computation of high order pseudospectral derivatives. Applied Numerical Mathematics 33, 151-159.

[15] Couzy, W., Deville, M. O., 1995. A Fast Schur Complement Method for the Spectral Element Discretization of the Incompressible Navier-Stokes Equations. J. Comput. Phys. 116, 135-142.

[16] Cros, J.-M., 2002. A preconditioner for the Schur complement domain decomposition method. 14th International Conference on Domain Decomposition Methods, 373-380.

[17] Diamessis, P. J., Domaradzki, J. A., Hesthaven, J. S., 2005. A spectral multidomain penalty method model for the simulation of high Reynolds number localized incompressible stratified turbulence. Journal of Computational Physics 202, 298-322.

[18] Diamessis, P. J., Redekopp, L. G., 2006. Numerical Investigation of Solitary Internal Wave-Induced Global Instability in Shallow Water Benthic Boundary Layers. Journal of Physical Oceanography 36, 784-812.

[19] Diamessis, P. J., Spedding, G. R., Domaradzki, J. A., 2011. Similarity scaling and vorticity structure in high-Reynoldsnumber stably stratified turbulent wakes. Journal of Fluid Mechanics 671, 52-95.

[20] Erlangga, Y. a., Nabben, R., 2008. Deflation and Balancing Preconditioners for Krylov Subspace Methods Applied to Nonsymmetric Matrices. SIAM Journal on Matrix Analysis and Applications 30, 684-699.

[21] Escobar-Vargas, J., Diamessis, P., Sakai, T., 2014. A spectral quadrilateral multidomain penalty method model for high Reynolds number incompressible stratified flows. International Journal for Numerical Methods in Fluids 75 (March), 403-425.

[22] Farhat, C., Lesoinne, M., Letallec, P., Pierson, K., Rixen, D., 2001. FETI-DP: A dual-primal unified FETI method part I: A faster alternative to the two-level FETI method. International Journal for Numerical Methods in Engineering 50 (August 1999), 1523-1544.

[23] Farhat, C., Roux, F.-X., 1991. A method of finite element tearing and interconnecting and its parallel solution algorithm. Int. J. Numer. Methods Engrg. 32 (February 1990), 1205-1227.

[24] Farhat, C., Tezaur, R., Toivanen, J., 2009. A domain decomposition method for discontinuous Galerkin discretizations of Helmholtz problems with plane waves and Lagrange multipliers. International Journal for Numerical Methods in Engineering 78, 1513-1531.

[25] Feng, X., Karakashian, O. A., 2001. Two-Level Additive Schwarz Methods for a Discontinuous Galerkin Approximation of Second Order Elliptic Problems. SIAM Journal on Numerical Analysis 39, 1343-1365.

[26] Fischer, P., 1997. An overlapping Schwarz method for spectral element solution of the incompressible Navier-Stokes equations. Journal of Computational Physics, 1-35. 
[27] Fischer, P. F., Lottes, J. W., 2005. Hybrid Schwarz-multigrid method for the spectral element method: extensions to Navier-Stokes. In: Domain Decomposition Methods in Science and Engineering. Springer Berlin Heidelberg, Ch. 1, p. 16.

[28] Gaul, A., Gutknecht, M., Liesen, J., Nabben, R., 2013. A Framework for Deflated and Augmented Krylov Subspace Methods. SIAM Journal on Matrix Analysis and Applications 34, 495-518.

[29] Geyer, W. R., Ralston, D. K., Hole, W., 2011. The Dynamics of Strongly Stratified Estuaries. Vol. 2. Elsevier Inc. URL http://dx.doi.org/10.1016/B978-0-12-374711-2.00206-0

[30] Ghysels, P., Ashby, T. J., Meerbergen, K., Vanroose, W., 2013. Hiding Global Communication Latency in the GMRES Algorithm on Massively Parallel Machines. SIAM Journal on Scientific Computing 35, C48-C71.

[31] Greenbaum, A., Pták, V., Strakoš, Z. k., 1996. Any Nonincreasing Convergence Curve is Possible for GMRES. SIAM Journal on Matrix Analysis and Applications 17 (3), 465-469.

[32] Hager, W. W., 1989. Updating the inverse of a matrix. SIAM Review 31 (2), 221-239.

[33] Hesthaven, J. S., 1997. A Stable Penalty Method for the Compressible Navier-Stokes Equations: II. One-Dimensional Domain Decomposition Schemes. SIAM Journal on Scientific Computing 18 (3), 658-685.

[34] Hesthaven, J. S., 1998. A Stable Penalty Method for the Compressible Navier-Stokes Equations: III. Multidimensional Domain Decomposition Schemes. SIAM Journal on Scientific Computing 20 (1), 62-93.

[35] Hesthaven, J. S., Gottlieb, S., Gottlieb, D., 2007. Spectral Methods for Time-Dependent Problems. Cambridge University Press.

[36] Hofmann, M., Kontoghiorghes, E. J., 2006. Pipeline Givens sequences for computing the QR decomposition on a EREW PRAM. Parallel Computing 32 (2006), 222-230. URL http://linkinghub.elsevier.com/retrieve/pii/S0167819105001638

[37] Kanschat, G., 2003. Preconditioning Methods for Local Discontinuous Galerkin Discretizations. SIAM Journal on Scientific Computing 25, 815-831.

[38] Karniadakis, G. E., 1989. Spectral element simulations of laminar and turbulent flows in complex geometries. Applied Numerical Mathematics 6, 85-105.

[39] Karniadakis, G. E., Israeli, M., Orszag, S. a., 1991. High-order splitting methods for the incompressible Navier-Stokes equations. Journal of Computational Physics 97, 414-443.

[40] Karypis, G., 2013. ParMETIS - Parallel Graph Partitioning and Fill-reducing Matrix Ordering. URL http://glaros.dtc.umn.edu/gkhome/metis/parmetis/overview

[41] Li, J., Widlund, O., 2006. BDDC Algorithms for Incompressible Stokes Equations. SIAM Journal on Numerical Analysis $44,2432-2455$.

[42] Lien, R.-C., DAsaro, E. a., Henyey, F., Chang, M.-H., Tang, T.-Y., Yang, Y.-J., 2012. Trapped Core Formation within a Shoaling Nonlinear Internal Wave. Journal of Physical Oceanography 42 (2004), 511-525. URL http://journals .ametsoc .org/doi/abs/10.1175/2011JP04578.1

[43] Manna, M., Vacca, A., Deville, M. O., 2004. Preconditioned spectral multi-domain discretization of the incompressible Navier-Stokes equations. Journal of Computational Physics 201, 204-223.

[44] Meurant, G., Duintjer Tebbens, J., 2014. The role eigenvalues play in forming GMRES residual norms with non-normal matrices. Numerical Algorithms, 143-165.

[45] Nabben, R., Vuik, C., 2004. A Comparison of Deflation and Coarse Grid Correction Applied to Porous Media Flow. SIAM Journal on Numerical Analysis 42 (0), 1631-1647.

[46] Nicolaides, R. a., 1987. Deflation of Conjugate Gradients with Applications to Boundary Value Problems. SIAM Journal on Numerical Analysis 24 (2), 355-365.

[47] Olson, L. N., Hesthaven, J. S., Wilcox, L. C., 2004. Developments in Overlapping Schwarz Preconditioning of High-Order Nodal Discontinuous Galerkin Discretizations. In: Domain Decomposition Methods in Science and Engineering XVI. No. November 2004. pp. 1-8.

[48] Pasquetti, R., Rapetti, F., Pavarino, L., Zampieri, E., 2006. Neumann-Neumann-Schur complement methods for Fekete spectral elements. Journal of Engineering Mathematics 56, 323-335.

[49] Pavarino, L. F., Warburton, T., 2000. Overlapping Schwarz Methods for Unstructured Spectral Elements. Journal of Computational Physics 160, 298-317.

[50] Pozrikidis, C., 2001. A Note on the Regularization of the Discrete PoissonNeumann Problem. Journal of Computational Physics 172, 917-923.

[51] Saad, Y., 2003. Iterative Methods for Sparse Linear Systems. Vol. 3. Society for Industrial and Applied Mathematics.

[52] Santilli, E., Scotti, A., 2011. An efficient method for solving highly anisotropic elliptic equations. Journal of Computational Physics 230 (23), 8342-8359.

[53] Santilli, E., Scotti, A., 2015. The Stratified Ocean Model with Adaptive Refinement. Journal of Computational Physics 291, 60-81.

[54] Scotti, A., Mitran, S., 2008. An approximated method for the solution of elliptic problems in thin domains: Application to nonlinear internal waves. Ocean Modelling 25, 144-153.

[55] Tang, J., Vuik, K., 2012. Acceleration of Preconditioned Krylov Solvers for Bubbly Flow Problems. In: Parallel Processing and Applied Mathematics. Vol. 7203. pp. 286-296.

[56] Tang, J. M., MacLachlan, S. P., Nabben, R., Vuik, C., 2010. A Comparison of Two-Level Preconditioners Based on Multigrid and Deflation. SIAM Journal on Matrix Analysis and Applications 31, 1715-1739.

[57] Tang, J. M., Nabben, R., Vuik, C., Erlangga, Y. A., 2009. Comparison of two-level preconditioners derived from deflation, domain decomposition and multigrid methods. Journal of Scientific Computing 39, 340-370.

[58] Toselli, A., Widlund, O., 2004. Domain Decomposition Methods - Algorithms and Theory. Springer.

[59] Vitousek, S., Fringer, O. B., 2011. Physical vs. numerical dispersion in nonhydrostatic ocean modeling. Ocean Modelling 40 (1), 72-86.

[60] Vitousek, S., Fringer, O. B., 2014. A nonhydrostatic, isopycnal-coordinate ocean model for internal waves. Ocean Modelling 83, 118-144.

[61] Vuik, C., Nabben, R., Tang, J., 2006. Deflation acceleration for domain decomposition preconditioners. In: Proceedings of the 8th European Multigrid Conference on Multigrid, Multilevel and Multiscale Methods. Vol. 29291. pp. 1-10. 
[62] Walker, H. F., 1988. Implementation of the GMRES Method Using Householder Transformations. SIAM Journal on Scientific and Statistical Computing 9 (1), 152-163.

[63] Watanabe, T., Riley, J. J., Kops, S. M. D. B., Diamessis, P. J., Zhou, Q., 2015. Turbulent / non-turbulent interfaces in wakes in stably-stratified fluids. Submitted to Journal of Fluid Mechanics.

[64] Yamazaki, I., Li, X. S., 2010. On techniques to improve robustness and scalability of the Schur complement method. In: Proceedings of the 9th International VECPAR Conference. No. 2.

URL http://vecpar.fe.up.pt/2010/papers/43.pdf

[65] Yamazaki, I., Li, X. S., Ng, E. G., 2010. Preconditioning Schur complement systems of highly-indefinite linear systems for a parallel hybrid solver. Numerical Mathematics 3, 352-366.

[66] Zhou, Q., Diamessis, P. J., 2013. Reflection of an internal gravity wave beam off a horizontal free-slip surface. Physics of Fluids 25 (May 2015). 